\title{
Identification of the glycan binding profile of human and rodent Plasmodium sporozoites.
}

Jessica Poole ${ }^{1 \#}$, Lauren E. Hartley-Tassell ${ }^{1 \#}$, Christopher J. Day ${ }^{1}$, Danielle I. Stanisic ${ }^{1}$, Penny L. Groves ${ }^{3}$, Sumana Chakravarty ${ }^{2}$, B. Kim Lee Sim ${ }^{2}$, Stephen L. Hoffman ${ }^{2}$, Joe Tiralongo ${ }^{1}$, Nicolai Bovin ${ }^{4}$, Denise L. Doolan ${ }^{5 *}$, and Michael P. Jennings ${ }^{1 *}$

${ }^{1}$ Institute for Glycomics, Griffith University, Southport 4222, Queensland, Australia.

${ }^{2}$ Sanaria Inc, Rockville, Maryland 20852, United States of America.

${ }^{3}$ QIMR Berghofer Medical Research Institute, Herston 4029, Queensland, Australia.

${ }^{4}$ Shemyakin Institute of Bioorganic Chemistry, Russian Academy of Sciences, Moscow, 117997, Russia.

${ }^{5}$ Centre for Molecular Therapeutics, Australian Institute of Tropical Health and Medicine, James Cook University, Cairns 4878, Queensland, Australia.

\# These authors contributed equally to this work.

*Correspondence:

Michael P. Jennings, Institute for Glycomics (G25), Griffith University Gold Coast, Southport, Parklands Dr, Queensland, 4222, Australia

Email: m.jennings@griffith.edu.au

Telephone: +61 755527050

Denise L. Doolan, Australian Institute of Tropical Health and Medicine, McGregor Road, Smithfield QLD 4878, Australia

Email: Denise.Doolan@jcu.edu.au

Telephone: +61 742321492 
Table S1. Glycan Array Results for P. falciparum and $P$. yoelii sporozoites.

\begin{tabular}{|c|c|c|c|c|}
\hline ID & Name & Structure & $\begin{array}{c}P . \\
\text { falciparum } \\
\text { Fold- } \\
\text { change }\end{array}$ & $\begin{array}{l}\text { P. yoelii } \\
\text { Fold- } \\
\text { change }\end{array}$ \\
\hline \multicolumn{5}{|c|}{ Terminal Gal } \\
\hline 2 & Gal $\alpha$ monosaccharide & Gal $\alpha$-sp3 & 4.34 & 1.51 \\
\hline 3 & Gal $\beta$ monosaccharide & Gal $\beta$-sp3 & - & - \\
\hline 37 & 3-O-Su- $\beta-\mathrm{Gal}$ & 3-O-Su-Galß-sp3 & 1.65 & - \\
\hline 75 & $\alpha 1-2$ Galactobiose & Gala1-2Gal $\beta$-sp3 & - & - \\
\hline 76 & a1-3 Galactobiose & Gala1-3Gal $\beta-$ sp3 & - & - \\
\hline 77 & $T \alpha \beta$ & Gal $\alpha 1-3$ GalNAc $\beta-\operatorname{sp} 3$ & 2.26 & - \\
\hline 78 & T $\alpha \alpha$ & Gal $\alpha 1-3$ GalNAc $\alpha-\operatorname{sp} 3$ & - & - \\
\hline 80 & $\mathrm{Aa} 3 \mathrm{GN}$ & Gal $\alpha 1-3$ GlcNAc $\beta$-sp3 & - & - \\
\hline 81 & $\alpha$-LacNAc & Gal $\alpha 1-4 \mathrm{GlcNAc} \beta-\mathrm{sp} 3$ & - & - \\
\hline 83 & Melibiose & Gal $\alpha 1-6 \mathrm{Glc} \beta$-sp4 & - & - \\
\hline 84 & $\beta 1-2$ Galactobiose & Gal $\beta 1-2 \mathrm{Gal} \beta-\mathrm{sp} 3$ & - & - \\
\hline 85 & $\mathrm{LeC}$ & Gal $\beta 1-3$ GlcNAc $\beta-s p 3$ & - & - \\
\hline 87 & $\beta 1-3$ Galactobiose & Gal $\beta 1-3 \mathrm{Gal} \beta-\mathrm{sp} 3$ & - & - \\
\hline 88 & $T \beta \beta$ & Gal $\beta 1-3$ GalNAc $\beta$-sp3 & - & - \\
\hline 89 & TF Antigen & Gal $\beta 1-3$ GalNAc $\alpha-s p 3$ & 1.81 & - \\
\hline 93 & Lactose & Gal $\beta 1-4$ Glc $\beta$-sp4 & - & - \\
\hline 94 & $\beta 1-4$ Galactobiose & Gal $\beta 1-4 \mathrm{Gal} \beta-\mathrm{sp} 4$ & - & - \\
\hline 97 & LacNAc & Gal $\beta 1-4$ GlcNAc $\beta$-sp3 & - & - \\
\hline 100 & $\beta 1-6$ Galactobiose & Gal $\beta 1-6 \mathrm{Gal} \beta-\mathrm{sp} 4$ & - & - \\
\hline 145 & 6-O-Su-LeC & Galß1-3(6-O-Su)GlcNAcß-sp3 & - & - \\
\hline 146 & 6-O-Su-Lactose & Gal $\beta 1-4(6-\mathrm{O}-\mathrm{Su}) \mathrm{Glc} \beta-\mathrm{sp} 2$ & - & - \\
\hline 147 & 6-O-Su-LacNAc & Galß1-4(6-O-Su)GlcNAcß-sp3 & - & - \\
\hline 150 & 3-O-Su-TF & 3-O-Su-Gal $\beta 1-3$ GalNAc $\alpha-$ sp3 & - & - \\
\hline 151 & 6-O-Su-TF Antigen & 6-O-Su-Gal $\beta 1-3$ GalNAc $\alpha-$ sp3 & - & 1.10 \\
\hline 152 & SM3 & 3-O-Su-Gal $\beta 1-4$ Glc $\beta$-sp2 & - & - \\
\hline 153 & 6-O-Su-Lactose & 6-O-Su-Gal $\beta 1-4 \mathrm{Glc} \beta-\mathrm{sp} 2$ & - & - \\
\hline 155 & 3`-O-Su-LeC & 3-O-Su-Gal $\beta 1-3$ GlcNAc $\beta$-sp3 & - & - \\
\hline 157 & 3`-O-Su-LacNAc & 3-O-Su-Gal $\beta 1-4$ GlcNAc $\beta$-sp3 & - & - \\
\hline 159 & 4-O-Su-LacNAc & 4-O-Su-Gal $\beta 1-4 \mathrm{GlcNAc} \beta$-sp3 & - & - \\
\hline 161 & 6-O-Su-LeC & 6-O-Su-Gal $\beta 1-3$ GlcNAc $\beta$-sp3 & - & - \\
\hline 163 & 6-O-Su-LacNAc & 6-O-Su-Gal $\beta 1-4 \mathrm{GlcNAc} \beta$-sp3 & - & - \\
\hline 176 & 3',6-di-O-Su-Lactose & $\begin{array}{l}\text { 3-O-Su-Gal } \beta 1-4(6-\mathrm{O}-\mathrm{Su}) \mathrm{Glc} \beta- \\
\mathrm{sp} 2\end{array}$ & - & - \\
\hline 177 & $\begin{array}{l}\text { 3’,6-di-O-Su- } \\
\text { LacNAc }\end{array}$ & $\begin{array}{l}\text { 3-O-Su-Gal } \beta 1-4(6-\mathrm{O}- \\
\text { Su)GlcNAc } \beta \text {-sp2 }\end{array}$ & - & - \\
\hline 178 & 6,6-di-O-Su-Lactose & $\begin{array}{l}\text { 6-O-Su-Gal } \beta 1-4(6-\mathrm{O}-\mathrm{Su}) \mathrm{Glc} \beta- \\
\mathrm{sp} 2\end{array}$ & - & - \\
\hline 179 & 6,6-di-O-Su-LeC & $\begin{array}{l}\text { 6-O-Su-Gal } \beta 1-3(6-\mathrm{O}- \\
\mathrm{Su}) \mathrm{GlcNAc} \beta-\mathrm{sp} 2\end{array}$ & - & - \\
\hline 180 & $\begin{array}{l}\text { 6,6-di-O-Su- } \\
\text { LacNAc }\end{array}$ & $\begin{array}{l}\text { 6-O-Su-Gal } \beta 1-4(6-\mathrm{O}- \\
\text { Su)GlcNAc } \beta \text {-sp2 }\end{array}$ & - & - \\
\hline
\end{tabular}




\begin{tabular}{|c|c|c|c|c|}
\hline 181 & $\begin{array}{l}\text { 3', } 4 \text {-di-O-Su- } \\
\text { LacNAc }\end{array}$ & $\begin{array}{c}\text { 3,4-O-Su2-Gal } \beta 1-4 G 1 c N A c \beta- \\
\text { sp3 }\end{array}$ & - & - \\
\hline 182 & $\begin{array}{l}3,6{ }^{\prime}-\mathrm{di}-\mathrm{O}-\mathrm{Su}- \\
\text { LacNAc }\end{array}$ & $\begin{array}{c}\text { 3,6-O-Su2-Gal } \beta 1-4 G l c N A c \beta- \\
\text { sp2 }\end{array}$ & - & - \\
\hline 183 & $\begin{array}{l}\text { 4,6`-di-O-Su- } \\
\text { LacNAc }\end{array}$ & $\begin{array}{c}\text { 4,6-O-Su2-Gal } \beta 1-4 \text { GlcNAc } \beta- \\
\text { sp2 }\end{array}$ & - & - \\
\hline 184 & $\begin{array}{l}4,6 `-d i-O-S u- \\
\text { LacNAc }\end{array}$ & $\begin{array}{l}\text { 4,6-O-Su2-Gal } \beta 1-4 \mathrm{GlcNAc} \beta- \\
\text { sp3 }\end{array}$ & - & - \\
\hline 189 & $\begin{array}{c}\text { 3`,6,6`-tri-O-Su- } \\
\text { LacNAc }\end{array}$ & $\begin{array}{c}\text { 3,6-O-Su2-Gal } \beta 1-4(6-\mathrm{O}- \\
\mathrm{Su}) \text { GlcNAc } \beta-\mathrm{sp} 2\end{array}$ & - & - \\
\hline 201 & $3,4-\mathrm{Su}_{2}$-LacdiNAc & $\begin{array}{c}\text { 3,4-O-Su2-Gal } \beta 1-4 G l c N A c \beta- \\
\text { sp3 }\end{array}$ & - & - \\
\hline 203 & 6-O-Su-LacNAc & Gal $\beta 1-4(6-\mathrm{O}-\mathrm{Su}) \mathrm{GlcNAc} \beta$-sp2 & - & - \\
\hline 220 & Aa3'Lac-C2 & Gal $\alpha 1-3$ Gal $\beta 1-4 \mathrm{Glc} \beta-\mathrm{sp} 2$ & - & 1.61 \\
\hline 222 & Galili/ $\alpha$ Gal epitope & Gal $\alpha 1-3$ Gal $\beta 1-4 G 1 c N A c \beta-s p 3$ & - & 1.81 \\
\hline 224 & $\mathrm{Pk}$ & Gal $\alpha 1-4 \mathrm{Gal} \beta 1-4 \mathrm{Glc} \beta-\mathrm{sp} 3$ & - & - \\
\hline 225 & P1 Antigen & Gal $\alpha 1-4 G a 1 \beta 1-4 G l c N A c-s p 2$ & - & - \\
\hline 228 & Ab2aLN & Gal $\beta 1-2$ Gal $\alpha 1-4$ GlcNAc $\beta$-sp4 & - & - \\
\hline 229 & Ab3`LN & Gal $\beta 1-3 \mathrm{Gal} \beta 1-4 \mathrm{GlcNAc} \beta-\mathrm{sp} 4$ & - & - \\
\hline 231 & LN3Tn & $\begin{array}{l}\text { Gal } \beta 1-4 G 1 c N A c \beta 1- \\
\text { 3GalNAc } \alpha-s p 3 \\
\end{array}$ & 1.52 & - \\
\hline 232 & LN6Tn & $\begin{array}{l}\text { Gal } \beta 1-4 \text { GlcNAc } \beta 1- \\
\text { 6GalNAc } \alpha-s p 3\end{array}$ & - & - \\
\hline 254 & Core 2 & $\begin{array}{l}\text { Gal } \beta 1-3(\text { GlcNAc } \beta 1- \\
\text { 6)GalNAc } \alpha-s p 3\end{array}$ & - & - \\
\hline 262 & $\mathrm{~T} \beta \beta-\mathrm{Gal}$ & Gal $\beta 1-3$ GalNAc $\beta 1-3$ Gal-sp4 & - & - \\
\hline 264 & Ab4'LN & Gal $\beta 1-4 G a 1 \beta 1-4 G l c N A c-s p 3$ & 3.97 & - \\
\hline 373 & Galili Antigen (tetra) & $\begin{array}{l}\text { Gal } \alpha 1-3 \text { Gal } \beta 1-4 G l c N A c \beta 1- \\
\text { 3Gal } \beta-\text { sp3 }\end{array}$ & - & - \\
\hline 375 & aLN3'LN & $\begin{array}{c}\text { Gal } \alpha 1-4 \text { GlcNAc } \beta 1-3 \text { Gal } \beta 1- \\
\text { 4GlcNAc } \beta-\operatorname{sp} 3\end{array}$ & - & - \\
\hline 276 & Lacto-N-tetraose & $\begin{array}{c}\text { Gal } \beta 1-3 \text { GlcNAc } \beta 1-3 \text { Gal } \beta 1- \\
\text { 4Glc } \beta \text {-sp4 }\end{array}$ & - & - \\
\hline 377 & LeC $\beta 33^{\prime} \mathrm{LeC}$ & $\begin{array}{c}\text { Gal } \beta 1-3 \text { GlcNAc } \beta 1-3 \mathrm{Gal} \beta 1- \\
\text { 3GlcNAc } \beta \text {-sp2 }\end{array}$ & - & - \\
\hline 378 & $\mathrm{LeC} a 3^{`} \mathrm{LN}$ & $\begin{array}{c}\text { Gal } \beta 1-3 \text { GlcNAc } \alpha 1-3 \text { Gal } \beta 1- \\
\text { 4GlcNAc } \beta \text {-sp3 }\end{array}$ & - & - \\
\hline 379 & $\mathrm{LeC} \beta 3^{`} \mathrm{LN}$ & $\begin{array}{c}\text { Gal } \beta 1-3 \text { GlcNAc } \beta 1-3 \text { Gal } \beta 1- \\
\text { 4GlcNAc } \beta \text {-sp3 }\end{array}$ & - & - \\
\hline 380 & LeC $a 6^{\prime} \mathrm{LN}$ & $\begin{array}{c}\text { Gal } \beta 1-3 \text { GlcNAc } \alpha 1-6 \text { Gal } \beta 1- \\
\text { 4GlcNAc } \beta \text {-sp2 }\end{array}$ & - & - \\
\hline 381 & $\mathrm{LeC} \beta 6^{\prime} \mathrm{LN}$ & $\begin{array}{c}\text { Gal } \beta 1-3 \text { GlcNAc } \beta 1-6 \mathrm{Gal} \beta 1- \\
\text { 4GlcNAc } \beta \text {-sp2 }\end{array}$ & - & - \\
\hline 382 & Asialo-GM1 & $\begin{array}{c}\text { Gal } \beta 1-3 \text { GalNAc } \beta 1-4 \text { Gal } \beta 1- \\
\text { 4Glc } \beta-\mathrm{sp} 3\end{array}$ & - & - \\
\hline 383 & Lacto- $N$-neotetraose & $\begin{array}{c}\text { Gal } \beta 1-4 G 1 c N A c \beta 1-3 \text { Gal } \beta 1- \\
4 \text { Glc } \beta-\mathrm{sp} 2\end{array}$ & - & - \\
\hline 385 & $\mathrm{i}$ & $\begin{array}{c}\text { Gal } \beta 1-4 \text { GlcNAc } \beta 1-3 \mathrm{Gal} \beta 1- \\
\text { 4GlcNAc } \beta \text {-sp3 }\end{array}$ & - & - \\
\hline
\end{tabular}




\begin{tabular}{|c|c|c|c|c|}
\hline 387 & LN $\beta 6^{\prime} \mathrm{LN}$ & $\begin{array}{c}\text { Gal } \beta 1-4 \text { GlcNAc } \beta 1-6 \mathrm{Gal} \beta 1- \\
\text { 4GlcNAc } \beta-\mathrm{sp} 2\end{array}$ & - & - \\
\hline 388 & LN $\beta 6 \mathrm{TF}$ & $\begin{array}{c}\text { Gal } \beta 1-3(\text { Gal } \beta 1-4 G l c N A c \beta 1- \\
\text { 6)GalNAc } \alpha-s p 3\end{array}$ & - & - \\
\hline 401 & LeC3LeC & $\begin{array}{c}\text { Gal } \beta 1-3 \mathrm{GlcNAc} \beta 1-3 \mathrm{Gal} \beta 1- \\
\text { 3GlcNAc } \beta \text {-sp3 }\end{array}$ & - & - \\
\hline 419 & $(3 ` \mathrm{SuLN}) 3 ` \mathrm{LN}$ & $\begin{array}{c}\text { 3-O-SuGal } \beta 1-4 G 1 c N A c \beta 1- \\
\text { 3Gal } \beta 1-4 G l c N A c \beta-s p 3\end{array}$ & - & - \\
\hline 420 & $\left(4{ }^{`} \mathrm{SuLN}\right) 3^{`} \mathrm{LN}$ & $\begin{array}{c}\text { 4-O-SuGal } \beta 1-4 G l c N A c \beta 1- \\
\text { 3Gal } \beta 1-4 G l c N A c \beta-s p 3\end{array}$ & - & - \\
\hline 481 & $\begin{array}{l}\text { Galili Antigen } \\
\text { (penta) }\end{array}$ & $\begin{array}{c}\text { Gal } \alpha 1-3 \mathrm{Gal} \beta 1-4 \mathrm{GlcNAc} \beta 1- \\
\text { 3Gal } \beta 1-4 \mathrm{Glc} \beta-\mathrm{sp} 4\end{array}$ & - & - \\
\hline 488 & LN2-3,6Tn & $\begin{array}{l}\text { Gal } \beta 1-4 G 1 c N A c \beta 1-3(G a l \beta 1- \\
\text { 4GlcNAc } \beta 1-6) \text { GalNAc } \alpha-s p 3\end{array}$ & - & - \\
\hline 489 & LN3`(GN6')LN & $\begin{array}{c}\text { Gal } \beta 1-4 G l c N A c \beta 1- \\
\text { 3(GlcNAc } \beta 1-6) \text { Gal } \beta 1- \\
\text { 4GlcNAc-sp2 }\end{array}$ & - & - \\
\hline 490 & LN6 (GN3')LN & $\begin{array}{c}\text { GlcNAc } \beta 1-3(\mathrm{Gal} \beta 1- \\
\text { 4GlcNAc } \beta 1-6) \text { Gal } \beta 1- \\
\text { 4GlcNAc } \beta \text {-sp2 }\end{array}$ & - & - \\
\hline 498 & $(\mathrm{LN}) 3$ & (Galß1-4GlcNAc $\beta 1-3) 3-s p 3$ & - & - \\
\hline 499 & I & $\begin{array}{c}\text { Gal } \beta 1-4 G l c N A c \beta 1-3(\mathrm{Gal} \beta 1- \\
\text { 4GlcNAc } \beta 1-6) \text { Gal } \beta 1- \\
\text { 4GlcNAc-sp2 }\end{array}$ & - & - \\
\hline 501 & Gb5 & $\begin{array}{c}\text { Gal } \beta 1-3 \text { GalNAc } \beta 1-3 \text { Gal } \alpha 1- \\
\text { 4Gal } \beta 1-4 \text { Glc } \beta-\mathrm{sp} 4\end{array}$ & - & - \\
\hline $1 \mathrm{~A}$ & Lacto- $N$-Biose I & Gal $\beta 1-3 G l c N A c$ & - & 1.03 \\
\hline $1 B$ & $\begin{array}{c}N \text {-Acetyllactosamine } \\
\text { (LacNAc) }\end{array}$ & Gal $\beta 1-4 G l c N A c$ & - & - \\
\hline $1 \mathrm{C}$ & $\beta 1-4$ Galactobiose & Gal $\beta 1-4 \mathrm{Gal}$ & - & - \\
\hline $1 \mathrm{D}$ & $\begin{array}{l}\beta-1-6 \text { Galactosyl- } N- \\
\text { acetyl glucosamine }\end{array}$ & Gal $\beta 1-6$ GlcNAc & - & - \\
\hline $1 \mathrm{E}$ & $\begin{array}{l}\beta-1-3 \text { Galactosyl- } N- \\
\text { acetyl galactosamine }\end{array}$ & Gal $\beta 1-3$ GalNAc & - & - \\
\hline $1 \mathrm{~F}$ & Asialo-GM1 & $\begin{array}{l}\text { Galb1-3GalNAc } \beta 1-4 \mathrm{Gal} \beta 1- \\
\text { 4Glc }\end{array}$ & - & - \\
\hline $1 \mathrm{G}$ & Lacto- $N$-tetraose & $\begin{array}{c}\text { Gal } \beta 1-3 \mathrm{GlcNAc} \beta 1-3 \mathrm{Gal} \beta 1- \\
\text { 4Glc }\end{array}$ & - & - \\
\hline $1 \mathrm{H}$ & Lacto- $N$-neotetraose & $\begin{array}{c}\text { Gal } \beta 1-4 G 1 c N A c \beta 1-3 \mathrm{Gal} \beta 1- \\
\text { 4Glc }\end{array}$ & - & - \\
\hline $1 \mathrm{I}$ & Lacto- $N$-neohexaose & $\begin{array}{c}\text { Gal } \beta 1-4 G l c N A c \beta 1-6(G a l \beta 1- \\
\text { 4GlcNAc } \beta 1-3) \text { Gal } \beta 1-4 \mathrm{Glc}\end{array}$ & - & - \\
\hline $1 \mathrm{~J}$ & Lacto- $N$-hexaose & $\begin{array}{c}\text { Gal } \beta 1-4 G l c N A c \beta 1-6(G a l \beta 1- \\
\text { 3GlcNAc } \beta 1-3) \text { Gal } \beta 1-4 \mathrm{Glc}\end{array}$ & - & - \\
\hline $1 \mathrm{~K}$ & Globotriose & 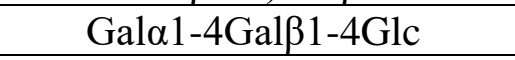 & - & - \\
\hline $1 \mathrm{M}$ & $\begin{array}{l}\text { Galactosyl-Tn } \\
\text { Antigen }\end{array}$ & Gal $\beta 1-3$ GalNAc $\alpha 1-O-S e r$ & - & - \\
\hline $1 \mathrm{~N}$ & $\alpha 1-3$ Galactobiose & Gala1-3Gal & - & - \\
\hline
\end{tabular}




\begin{tabular}{|c|c|c|c|c|}
\hline 10 & $\begin{array}{c}\text { Linear B-2 } \\
\text { Trisaccharide }\end{array}$ & Gal $\alpha 1-3$ Gal $\beta 1-4 G l c N A c$ & - & - \\
\hline $1 \mathrm{P}$ & $\begin{array}{c}\text { Linear B-6 } \\
\text { Trisaccharide }\end{array}$ & Gal $\alpha 1-3 \mathrm{Gal} \beta 1-4 \mathrm{Glc}$ & - & - \\
\hline $2 \mathrm{~A}$ & $\begin{array}{l}\alpha 1-3, \beta 1-4, \alpha 1-3 \\
\text { Galactotetraose }\end{array}$ & Gal $\alpha 1-3 \mathrm{Gal} \beta 1-4 \mathrm{Gal} \alpha 1-3 \mathrm{Gal}$ & - & - \\
\hline $2 \mathrm{~B}$ & $\beta 1-6$ Galactobiose & Gal $\beta 1-6 \mathrm{Gal}$ & - & - \\
\hline $2 \mathrm{D}$ & P1 Antigen & Gal $\alpha 1-4 G a 1 \beta 1-4 G l c N A c$ & - & - \\
\hline $2 \mathrm{G}$ & $\begin{array}{l}\text { iso-Lacto-N-octaose } \\
\text { (iLNO) }\end{array}$ & $\begin{array}{c}\text { Gal } \beta 1-3 \mathrm{GlcNAc} \beta 1-3 \mathrm{Gal} \beta 1- \\
\text { 4GlcNAc } \beta 1-6(\mathrm{Gal} \beta 1- \\
\text { 3GlcNAc } \beta 1-3) \text { Gal } \beta 1-4 \mathrm{Glc}\end{array}$ & - & - \\
\hline $2 \mathrm{H}$ & $\begin{array}{c}\text { para-Lacto-N- } \\
\text { hexaose (pLNH) }\end{array}$ & $\begin{array}{l}\text { Gal } \beta 1-3 \mathrm{GlcNAc} \beta 1-3 \mathrm{Gal} \beta 1- \\
4 \mathrm{GlcNAc} \beta 1-3 \mathrm{Gal} \beta 1-4 \mathrm{Glc} \\
\end{array}$ & - & - \\
\hline \multicolumn{5}{|c|}{ Terminal GlcNAc } \\
\hline 10 & $\beta$-GlcNAc & GlcNAc $\beta$-sp3 & - & 1.30 \\
\hline 22 & $\beta$-GlcNAc & GlcNAc $\beta$-sp4 & 2.07 & - \\
\hline 43 & 6-O-Su- $\beta-$ GlcNAc & 6-O-Su-GlcNAc $\beta-$ sp3 & 1.60 & - \\
\hline 55 & 3-O-Su- $\beta-\mathrm{GlcNAc}$ & 3-O-Su-GlcNAc $\beta$-sp3 & 1.91 & - \\
\hline 113 & Core 3 & GlcNAc $\beta 1-3$ GalNAc $\alpha-\operatorname{sp} 3$ & 2.87 & - \\
\hline 114 & Chitobiose-Asn & GlcNAc $\beta 1-4 G l c N A c \beta-A s n$ & - & 1.40 \\
\hline 115 & Chitobiose & GlcNAc $\beta 1-4 G l c N A c \beta-s p 4$ & 2.60 & 2.23 \\
\hline 117 & Core 6 & GlcNAc $\beta 1-6$ GalNAc $\alpha-$ sp3 & - & 2.07 \\
\hline 118 & 6-O-Su-chitobiose & $\begin{array}{l}\text { GlcNAc } \beta 1-4(6-\mathrm{O}- \\
\mathrm{Su}) \text { GlcNAc } \beta-\mathrm{sp} 2\end{array}$ & - & - \\
\hline 149 & GlcNAc-Mur & $\begin{array}{c}\text { GlcNAc } \beta 1-4- \\
\text { [HOOC(CH3)CH]-3-O- } \\
\text { GlcNAc } \beta \text {-sp4 }\end{array}$ & - & - \\
\hline 167 & GMDP-Lys & $\begin{array}{c}\text { GlcNAc } \beta 1-- \\
\text { [HOOC }(\mathrm{CH} 3) \mathrm{CH}]-3-\mathrm{O}- \\
\text { GlcNAc } \beta-\mathrm{L}-\text { alanyl-D-i- } \\
\text { glutaminyl-L-lysine }\end{array}$ & - & - \\
\hline 168 & GN2`TF & $\begin{array}{l}\text { GlcNAc } \beta 1-2 \text { Gal } \beta 1- \\
\text { 3GalNAc } \alpha \text {-sp3 }\end{array}$ & - & - \\
\hline 246 & GN3`TF & $\begin{array}{l}\text { GlcNAc } \beta 1-3 \text { Gal } \beta 1- \\
\text { 3GalNAc } \alpha-s p 3\end{array}$ & 2.79 & - \\
\hline 247 & GN3'Lac & GlcNAc $\beta 1-3$ Gal $\beta 1-4$ Glc $\beta$-sp2 & - & - \\
\hline 248 & GN3`LN & $\begin{array}{l}\text { GlcNAc } \beta 1-3 \mathrm{Gal} \beta 1- \\
\text { 4GlcNAc } \beta-\mathrm{sp} 3\end{array}$ & 4.19 & - \\
\hline 250 & GN4 LN & $\begin{array}{l}\text { GlcNAc } \beta 1-4 \mathrm{Gal} \beta 1- \\
\text { 4GlcNAc } \beta \text {-sp } 2\end{array}$ & - & - \\
\hline 251 & Chitotriose & $\begin{array}{c}\text { GlcNAc } \beta 1-4 G l c N A c \beta 1- \\
\text { 4GlcNAc } \beta \text {-sp4 }\end{array}$ & - & - \\
\hline 252 & GN6 ${ }^{\prime L N}$ & $\begin{array}{c}\text { GlcNAc } \beta 1-6 \mathrm{Gal} \beta 1- \\
\text { 4GlcNAc } \beta-\mathrm{sp} 2\end{array}$ & - & - \\
\hline 253 & Core 4 & $\begin{array}{c}\text { GlcNAc } \beta 1-3(\text { GlcNAc } \beta 1- \\
\text { 6)GalNAc } \alpha \text {-sp3 }\end{array}$ & - & - \\
\hline 255 & Tk & $\begin{array}{l}\text { GlcNAc } \beta 1-3(\text { GlcNAc } \beta 1- \\
\text { 6)Gal } \beta 1-4 G l c N A c \beta-s p 3\end{array}$ & - & - \\
\hline 395 & Chitopentaose & $($ GlcNAc $\beta 1-4) 5 \beta-s p 4$ & - & - \\
\hline
\end{tabular}




\begin{tabular}{|c|c|c|c|c|}
\hline 493 & Chitohexaose & (GlcNAc $\beta 1-4) 6 \beta-\mathrm{sp} 4$ & - & - \\
\hline 503 & $9-\mathrm{OS}$ & $\begin{array}{c}(\mathrm{A}-\mathrm{GN}-\mathrm{M}) 2-3,6-\mathrm{M}-\mathrm{GN}-\mathrm{GN} \beta- \\
\text { sp4 }\end{array}$ & - & - \\
\hline 504 & 7-OS & (GN-M)2-3,6-M-GN-GN $\beta$-sp4 & - & - \\
\hline 505 & $\begin{array}{l}N, N \text {-Diacetyl } \\
\text { chitobiose }\end{array}$ & GlcNAc $\beta 1-4 G l c N A c$ & - & - \\
\hline $4 \mathrm{~A}$ & $\begin{array}{l}N, N^{\prime}, N^{\prime \prime} \text {-Triacetyl } \\
\text { chitotriose }\end{array}$ & $\begin{array}{c}\text { GlcNAc } \beta 1-4 G l c N A c \beta 1- \\
\text { 4GlcNAc }\end{array}$ & - & - \\
\hline $4 \mathrm{~B}$ & $\begin{array}{l}N, N^{\prime}, N^{\prime \prime}, N^{\prime \prime}- \\
\text { Tetraacetyl } \\
\text { chitotetraose }\end{array}$ & $\begin{array}{l}\text { GlcNAc } \beta 1-4 G 1 c N A c \beta 1- \\
\text { 4GlcNAc } \beta 1-4 G l c N A c\end{array}$ & 1.84 & - \\
\hline $4 \mathrm{C}$ & $\begin{array}{c}N, N^{\prime}, N^{\prime \prime}, N^{\prime \prime \prime}, N^{\prime \prime \prime}, N^{\prime \prime \prime \prime-} \\
\text { Hexaacetyl } \\
\text { chitohexaose }\end{array}$ & $\begin{array}{c}\text { GlcNAc } \beta 1-4 G l c N A c \beta 1- \\
\text { 4GlcNAc } \beta 1-4 G l c N A c \beta 1- \\
\text { 4GlcNAc } \beta 1-4 G l c N A c\end{array}$ & - & - \\
\hline $4 \mathrm{D}$ & GlcNAc $\beta 1-4$ MurNAc & GlcNAc $\beta 1-4$ MurNAc & - & - \\
\hline $4 \mathrm{E}$ & $\begin{array}{c}\text { Pentacetyl } \\
\text { chitopentaose }\end{array}$ & $\begin{array}{l}\text { GlcNAc } \beta 1-4 G l c N A c \beta 1- \\
\text { 4GlcNAc } \beta 1-4 G l c N A c \beta 1- \\
\text { 4GlcNAc }\end{array}$ & - & - \\
\hline \multicolumn{5}{|c|}{ Mannosyl } \\
\hline 16 & $\alpha$-Man & Man $\alpha-s p 3$ & 2.32 & - \\
\hline 18 & $\beta$-Man & Man $\beta$-sp4 & - & - \\
\hline 19 & $\beta-\operatorname{ManAc}$ & ManNAc $\beta$-sp4 & - & - \\
\hline 47 & $\alpha-$ Man6P & 6-H2PO3Man $\alpha-s p 3$ & - & - \\
\hline 114 & GN3M & GlcNAc $\beta 1-3 M a n \beta-s p 4$ & 3.26 & - \\
\hline 119 & $\alpha 1-2$ Mannobiose & Man $\alpha 1-2$ Man $\beta$-sp4 & - & 1.21 \\
\hline 120 & $\alpha 1-3$ Mannobiose & Man $\alpha 1-3 \operatorname{Man} \beta-\mathrm{sp} 4$ & - & 1.82 \\
\hline 121 & $\alpha 1-4$ Mannobiose & Man $\alpha 1-4$ Man $\beta$-sp4 & - & - \\
\hline 122 & a1-6 Mannobiose & Man $\alpha 1-6 \operatorname{Man} \beta$-sp4 & - & - \\
\hline 123 & $\mathrm{Mb} 4 \mathrm{GN}$ & Man $\beta 1-4 G l c N A c \beta-s p 4$ & - & - \\
\hline 124 & $\alpha 1-2, \alpha$ Mannobiose & Man $\alpha 1-2$ Man $\alpha$-sp4 & - & - \\
\hline 258 & Man3 & Man $\alpha 1-3(\operatorname{Man} \alpha 1-6) \operatorname{Man} \beta-\operatorname{sp} 4$ & - & - \\
\hline 495 & $\begin{array}{l}\alpha 1-3, \alpha 1-3, \alpha 1-6- \\
\text { Mannopentaose }\end{array}$ & $\begin{array}{l}\text { Man } \alpha 1-6(\text { Man } \alpha 1-3) \text { Man } \alpha 1- \\
\text { 6(Man } \alpha 1-3) \text { Man } \beta-\operatorname{sp} 4\end{array}$ & - & - \\
\hline $5 \mathrm{~A}$ & $\begin{array}{c}\beta 1-2 N- \\
\text { Acetylglucosamine- } \\
\text { mannose }\end{array}$ & GlcNAc $\beta 1-2$ Man & - & - \\
\hline $5 \mathrm{~B}$ & $\begin{array}{l}\text { Bianntennary } N- \\
\text { linked core } \\
\text { pentasaccharide }\end{array}$ & $\begin{array}{c}\text { GlcNAc } \beta 1-2 \text { Man } \alpha 1- \\
\text { 6(GlcNAc } \beta 1-2 \text { Man } \alpha 1-3) \text { Man }\end{array}$ & - & - \\
\hline $5 \mathrm{C}$ & $\alpha 1-2-M a n n o b i o s e$ & Man $\alpha 1-2 M a n$ & - & - \\
\hline $5 \mathrm{D}$ & $\alpha 1-3-M a n n o b i o s e$ & Man $\alpha 1-3$ Man & - & - \\
\hline $5 \mathrm{E}$ & $\alpha 1-4-M a n n o b i o s e$ & Man 1 1-4Man & - & - \\
\hline $5 \mathrm{~F}$ & $\alpha 1-6-M a n n o b i o s e$ & Man $\alpha 1-6$ Man & - & - \\
\hline $5 \mathrm{G}$ & $\begin{array}{l}\alpha 1-3, \alpha 1-6- \\
\text { Mannobiose }\end{array}$ & Man $\alpha 1-6($ Man $\alpha 1-3)$ Man & - & - \\
\hline $5 \mathrm{H}$ & $\begin{array}{l}\alpha 1-3, \alpha 1-3, \alpha 1-6- \\
\text { Mannopentaose }\end{array}$ & $\begin{array}{l}\text { Man } 1 \text { 1-6(Man } \alpha 1-3) \text { Man } \alpha 1- \\
\text { 6(Man } 1 \text { 1-3) Man }\end{array}$ & - & - \\
\hline \multicolumn{5}{|c|}{ Fucosyl } \\
\hline 1 & L- $\alpha$-Fuc & Fuc $\alpha-\operatorname{sp} 3$ & - & - \\
\hline
\end{tabular}




\begin{tabular}{|c|c|c|c|c|}
\hline 71 & Blood Group H (di) & Fuc $\alpha 1-2$ Gal $\beta$-sp3 & - & - \\
\hline 72 & Fa3GN & Fuc $\alpha 1-3$ GlcNAc $\beta-s p 3$ & - & - \\
\hline 73 & Lewis core & Fuc $\alpha 1-4 G 1 c N A c \beta-s p 3$ & - & - \\
\hline 215 & Lewis D & Fuc $\alpha 1-2$ Gal $\beta 1-3$ GlcNAc $\beta$-sp3 & - & - \\
\hline 216 & $\begin{array}{l}\text { Blood Group H (type } \\
\text { 2) }\end{array}$ & Fuc $\alpha 1-2$ Gal $\beta 1-4 G 1 c N A c \beta-s p 3$ & 1.78 & - \\
\hline 217 & $\begin{array}{l}\text { Blood Group H (type } \\
3 \text { ) }\end{array}$ & Fuc $\alpha 1-2$ Gal $\beta 1-3$ GalNAc $\alpha-s p 3$ & - & - \\
\hline 219 & $\begin{array}{l}\text { Blood Group H (type } \\
6 \text { ) }\end{array}$ & Fuc $\alpha 1-2$ Gal $\beta 1-4$ Glc $\beta-$ sp 4 & - & - \\
\hline 226 & Blood Group B (tri) & Fuc $\alpha 1-2($ Gal $\alpha 1-3)$ Gal $\beta$-sp3 & - & - \\
\hline 233 & Lewis A & $\begin{array}{l}\text { Gal } \beta 1-3(\text { Fuc } \alpha 1-4) \text { GlcNAc } \beta- \\
\text { sp3 }\end{array}$ & 3.28 & - \\
\hline 234 & Lewis X & $\begin{array}{l}\text { Fuc } \alpha 1-3(\text { Gal } \beta 1-4) \text { GleNAc } \beta- \\
\text { sp3 }\end{array}$ & 4.11 & - \\
\hline 235 & Blood Group A (tri) & $\begin{array}{l}\text { Fuc } \alpha 1-2(\text { GalNAc } \alpha 1-3) \text { Gal } \beta- \\
\text { sp3 }\end{array}$ & - & - \\
\hline 287 & Su-Lewis A & $\begin{array}{l}\text { 3-O-Su-Gal } \beta 1-3(\text { Fuc } \alpha 1- \\
\text { 4)GlcNAc } \beta \text {-sp3 }\end{array}$ & - & - \\
\hline 288 & Su-Lewis X & $\begin{array}{c}\text { Fuc } \alpha 1-3(3-\mathrm{O}-\mathrm{Su}-\mathrm{Gal} \beta 1- \\
\text { 4)GlcNAc } \beta-\mathrm{sp} 3\end{array}$ & - & - \\
\hline 359 & $\begin{array}{l}\text { Blood Group B (type } \\
1 \text { ) }\end{array}$ & $\begin{array}{c}\text { Fuc } \alpha 1-2(\text { Gal } \alpha 1-3) \text { Gal } \beta 1- \\
\text { 3GlcNAc } \beta \text {-sp3 }\end{array}$ & - & - \\
\hline 360 & $\begin{array}{l}\text { Blood Group B (type } \\
\text { 2) }\end{array}$ & $\begin{array}{c}\text { Fuc } \alpha 1-2(\text { Gal } \alpha 1-3) \text { Gal } \beta 1- \\
\text { 4GlcNAc } \beta \text {-sp3 }\end{array}$ & - & - \\
\hline 362 & $\begin{array}{l}\text { Blood Group B (type } \\
3 \text { ) }\end{array}$ & $\begin{array}{c}\text { Fuc } \alpha 1-2(\text { Gal } \alpha 1-3) \text { Gal } \beta 1- \\
\text { 3GalNAc } \alpha-s p 3\end{array}$ & - & - \\
\hline 363 & $\begin{array}{l}\text { Blood Group B (type } \\
4 \text { ) }\end{array}$ & $\begin{array}{c}\text { Fuc } \alpha 1-2(\text { Gal } \alpha 1-3) \text { Gal } \beta 1- \\
\text { 3GalNAc } \beta \text {-sp3 } \\
\end{array}$ & - & - \\
\hline 364 & $\alpha$ Gal Lewis X & $\begin{array}{l}\text { Fuc } \alpha 1-3(\text { Gal } \alpha 1-3 \text { Gal } \beta 1- \\
\text { 4)GlcNAc } \beta-s p 3\end{array}$ & - & - \\
\hline 366 & $\begin{array}{l}\text { Blood Group A (type } \\
1 \text { ) }\end{array}$ & $\begin{array}{c}\text { Fuc } \alpha 1-2(\text { GalNAc } \alpha 1-3) \text { Gal } \beta 1- \\
\text { 3GlcNAc } \beta-s p 3\end{array}$ & - & - \\
\hline 368 & $\begin{array}{l}\text { Blood Group A (type } \\
\text { 2) }\end{array}$ & $\begin{array}{c}\text { Fuc } \alpha 1-2(\text { GalNAc } \alpha 1-3) \text { Gal } \beta 1- \\
\text { 4GlcNAc } \beta \text {-sp3 }\end{array}$ & - & - \\
\hline 371 & Lewis B & $\begin{array}{c}\text { Fuc } \alpha 1-2 \text { Gal } \beta 1-3 \text { (Fuc } \alpha 1- \\
\text { 4)GlcNAc } \beta \text {-sp3 }\end{array}$ & - & - \\
\hline 372 & Lewis Y & $\begin{array}{l}\text { Fuc } \alpha 1-3(\text { Fuc } \alpha 1-2 \text { Gal } \beta 1- \\
\text { 4)GlcNAc } \beta \text {-sp3 }\end{array}$ & - & - \\
\hline 392 & $\begin{array}{l}\text { Blood Group A (type } \\
3 \text { ) }\end{array}$ & $\begin{array}{l}\text { Fuc } \alpha 1-2 \text { (GalNAc } 1 \text { 1- } \\
\text { 6)GalNAc } \alpha 1-6-\text { sp3 }\end{array}$ & - & - \\
\hline 479 & LNFP-I & $\begin{array}{c}\text { Fuc } \alpha 1-2 \mathrm{Gal} \beta 1-3 \mathrm{GlcNAc} \beta 1- \\
\text { 3Gal } \beta 1-4 \mathrm{Glc} \beta-\mathrm{sp} 4\end{array}$ & - & - \\
\hline 480 & $\begin{array}{c}\text { Blood Group H (type } \\
1 ; \text { penta) }\end{array}$ & $\begin{array}{c}\text { Fuc } \alpha 1-2 \text { Gal } \beta 1-3 \text { GlcNAc } \beta 1- \\
\text { 3Gal } \beta 1-4 \text { GlcNAc } \beta-\mathrm{sp} 2\end{array}$ & - & - \\
\hline 483 & BLeY & $\begin{array}{c}\text { Gal } \alpha 1-3 \text { (Fuc } \alpha 1-2) \text { Gal } \beta 1-4 \\
\text { (Fuc } \alpha 1-3) \text { GlcNAc } \beta \text {-sp3 }\end{array}$ & - & - \\
\hline
\end{tabular}




\begin{tabular}{|c|c|c|c|c|}
\hline 496 & Lewis B-Lactose & $\begin{array}{l}\text { Fuc } \alpha 1-2 \text { Gal } \beta 1-3(\text { Fuc } \alpha 1- \\
\text { 4)GlcNAc } \beta 1-3 \text { Gal } \beta 1-4 G l c \beta- \\
\text { sp4 }\end{array}$ & - & - \\
\hline 497 & Lewis Y-Lactose & $\begin{array}{c}\text { Fuc } \alpha 1-3(\text { Fuc } \alpha 1-2 \text { Gal } \beta 1- \\
\text { 4)GlcNAc } \beta 1-3 \text { Gal } \beta 1-4 \text { Glc } \beta- \\
\text { sp4 }\end{array}$ & - & - \\
\hline 538 & MFLNH III & Lex1-6'(Lec1-3')Lac-sp4 & - & - \\
\hline 539 & MFLNH I & LacNAc1-6'(Led1-3')Lac-sp4 & - & - \\
\hline 541 & DFLNH (a) & Lex1-6'(Led1-3')Lac-sp4 & - & - \\
\hline 542 & MF(1-3)iLNO & LecLex1-6'(Lec1-3')Lac-sp4 & - & - \\
\hline 543 & TFLNH & Lex1-6'(Leb1-3')Lac-sp4 & - & - \\
\hline $7 \mathrm{~A}$ & $\begin{array}{c}\text { Lacto-N- } \\
\text { fucopentaose I }\end{array}$ & $\begin{array}{l}\text { Fuc } \alpha 1-2 \text { Gal } \beta 1-3 \text { GlcNAc } \beta 1- \\
\text { 3Gal } \beta 1-4 \text { Glc }\end{array}$ & - & - \\
\hline $7 \mathrm{~B}$ & $\begin{array}{c}\text { Lacto-N- } \\
\text { fucopentaose II }\end{array}$ & $\begin{array}{c}\text { Gal } \beta 1-3(\text { Fuc } \alpha 1-4) \text { GlcNAc } \beta 1- \\
\text { 3Gal } \beta 1-4 \text { Glc }\end{array}$ & - & - \\
\hline $7 \mathrm{C}$ & $\begin{array}{c}\text { Lacto-N- } \\
\text { fucopentaose III }\end{array}$ & $\begin{array}{c}\text { Gal } \beta 1-4(\text { Fuc } \alpha 1-3) \text { GlcNAc } \beta 1- \\
\text { 3Gal } \beta 1-4 \text { Glc }\end{array}$ & - & - \\
\hline $7 \mathrm{D}$ & $\begin{array}{c}\text { Lacto-N- } \\
\text { difucohexaose I }\end{array}$ & $\begin{array}{l}\text { Fuc } \alpha 1-2 \text { Gal } \beta 1-3 \text { (Fuc } \alpha 1- \\
\text { 4)GlcNAc } \beta 1-3 \text { Gal } \beta 1-4 G l c\end{array}$ & - & - \\
\hline $7 \mathrm{E}$ & $\begin{array}{c}\text { Lacto-N- } \\
\text { difucohexaose II }\end{array}$ & $\begin{array}{c}\text { Gal } \beta 1-3(\text { Fuc } \alpha 1-4) \text { GlcNAc } \beta 1- \\
\text { 3Gal } \beta 1-4(\text { Fuc } \alpha 1-3) \text { Glc }\end{array}$ & - & - \\
\hline $7 \mathrm{~F}$ & Blood Group H (di) & Fuc $\alpha 1-2 \mathrm{Gal}$ & - & - \\
\hline $7 \mathrm{G}$ & 2'-Fucosyllactose & Fuc $\alpha 1-2$ Gal $\beta 1-4$ Glc & - & - \\
\hline $7 \mathrm{H}$ & 3'-Fucosyllactose & Gal $\beta 1-4($ Fuc $\alpha 1-3)$ Glc & - & - \\
\hline $7 \mathrm{I}$ & Lewis X & Gal $\beta 1-4($ Fuc $\alpha 1-3)$ GlcNAc & - & - \\
\hline $7 \mathrm{~J}$ & Lewis A & Gal $\beta 1-3($ Fuc $\alpha 1-4)$ GlcNAc & - & - \\
\hline $7 \mathrm{~K}$ & Blood Group A (tri) & GalNAc $\alpha 1-3($ Fuc $\alpha 1-2)$ Gal & - & - \\
\hline $7 \mathrm{~L}$ & $\begin{array}{l}\text { Lactodifucotetraose } \\
\text { (LDFT) }\end{array}$ & Fuc $\alpha 1-2$ Gal $\beta 1-4($ Fuc $\alpha 1-3)$ Glc & - & - \\
\hline $7 \mathrm{M}$ & Blood Group B (tri) & Gal $\alpha 1-3($ Fuc $\alpha 1-2)$ Gal & - & - \\
\hline $7 \mathrm{~N}$ & Lewis Y & $\begin{array}{c}\text { Fuc } \alpha 1-2 \text { Gal } \beta 1-4 \text { (Fuc } \alpha 1- \\
\text { 3)GlcNAc }\end{array}$ & - & - \\
\hline 70 & $\begin{array}{c}\text { Blood Group H (type } \\
2 ; \text { tri) }\end{array}$ & Fuc $\alpha 1-2$ Gal $\beta 1-3$ GlcNAc & - & - \\
\hline $7 \mathrm{P}$ & Lewis B (tetra) & $\begin{array}{c}\text { Fuc } \alpha 1-2 \text { Gal } \beta 1-3 \text { (Fuc } \alpha 1- \\
\text { 4)GlcNAc }\end{array}$ & - & - \\
\hline $8 \mathrm{~A}$ & Su-Lewis A & $\begin{array}{c}\text { SO3-3Gal } \beta 1-3 \text { (Fuc } \alpha 1- \\
\text { 4)GlcNAc }\end{array}$ & - & - \\
\hline $8 \mathrm{~B}$ & Su-Lewis X & $\begin{array}{l}\text { SO3-3Gal } \beta 1-4(\text { Fuc } \alpha 1- \\
\text { 3)GlcNAc }\end{array}$ & - & - \\
\hline $8 \mathrm{C}$ & $\begin{array}{l}\text { Monofucosyl-para- } \\
\text { Lacto-N-hexaose IV }\end{array}$ & $\begin{array}{c}\text { Gal } \beta 1-3 \text { GlcNAc } \beta 1-3 \text { Gal } \beta 1- \\
\text { 4(Fuc } \alpha 1-3) \text { GlcNAc } \beta 1-3 \text { Gal } \beta 1- \\
\text { 4Glc }\end{array}$ & - & - \\
\hline $8 \mathrm{D}$ & $\begin{array}{l}\text { Monofucosyllacto-N- } \\
\text { hexaose III }\end{array}$ & $\begin{array}{c}\text { Gal } \beta 1-4(\text { Fuc } \alpha 1-3) \text { GlcNAc } \beta 1- \\
\text { 6(Gal } \beta 1-3 \text { GlcNAc } \beta 1-3) \text { Gal } \beta 1- \\
\text { 4Glc }\end{array}$ & - & - \\
\hline $8 \mathrm{E}$ & $\begin{array}{l}\text { Difucosyllacto-N- } \\
\text { hexaose }\end{array}$ & $\begin{array}{c}\text { Gal } \beta 1-4(\text { Fuc } \alpha 1-3) \text { GlcNAc } \beta 1- \\
\text { 6(Fuc } \alpha 1-2 \text { Gal } \beta 1-3 \text { GlcNAc } \beta 1- \\
\text { 3)Gal } \beta 1-4 \text { Glc }\end{array}$ & - & - \\
\hline
\end{tabular}




\begin{tabular}{|c|c|c|c|c|}
\hline $8 \mathrm{~F}$ & $\begin{array}{l}\text { Trifucosyllacto-N- } \\
\text { hexaose }\end{array}$ & $\begin{array}{c}\text { Gal } \beta 1-4(\text { Fuc } \alpha 1-3) \text { GlcNAc } \beta 1- \\
\text { 6(Fuc } \alpha 1-2 \text { Gal } \beta 1-3(\text { Fuc } \alpha 1- \\
\text { 4)GlcNAc } \beta 1-3) \text { Gal } \beta 1-4 G l c\end{array}$ & - & - \\
\hline $8 \mathrm{G}$ & $\begin{array}{c}\text { Lacto-N- } \\
\text { fucopentaose VI } \\
(\text { LNFP VI) }\end{array}$ & $\begin{array}{c}\text { Gal } \beta 1-4 G l c N A c \beta 1-3 G a l \beta 1- \\
\text { 4(Fuc } \alpha 1-3) \text { Glc }\end{array}$ & - & - \\
\hline $8 \mathrm{H}$ & $\begin{array}{l}\text { Lacto-N- } \\
\text { neodifucohexaose I } \\
(\mathrm{LNnDFH} \mathrm{I)}\end{array}$ & $\begin{array}{l}\text { Fuc } \alpha 1-2 \text { Gal } \beta 1-4(\text { Fuc } \alpha 1- \\
\text { 3)GlcNAc } \beta 1-3 \text { Gal } \beta 1-4 G 1 c\end{array}$ & - & - \\
\hline $8 \mathrm{I}$ & $\begin{array}{c}\text { Lacto-N- } \\
\text { neodifucohexaose II } \\
(\mathrm{LNnDFH} \mathrm{II)} \\
\end{array}$ & $\begin{array}{l}\text { Fuc } \alpha 1-3 \mathrm{Gal} \beta 1-4 \mathrm{GlcNAc} \beta 1- \\
\text { 3Gal } \beta 1-4(\text { Fuc } \alpha 1-3) \text { Glc }\end{array}$ & - & 2.10 \\
\hline $8 \mathrm{~J}$ & $\begin{array}{l}\text { Trifucosyllacto-N- } \\
\text { neoteraose I } \\
\text { (TFLNnTI) }\end{array}$ & $\begin{array}{l}\text { Fuc } \alpha 1-2 \text { Galb1-4(Fuc } \alpha 1- \\
\text { 3)GlcNAcb1-3(Fuc } 1 \text { 1- } \\
\text { 2)Galb1-4Glc }\end{array}$ & - & - \\
\hline $8 \mathrm{~K}$ & $\begin{array}{l}\text { Monofucosyllacto- } \\
\text { N-neohexaose I } \\
\text { (MFLNnH I) }\end{array}$ & $\begin{array}{c}\text { Gal } \beta 1-4(\text { Fuc } \alpha 1-3) \text { GlcNAc } \beta 1- \\
\text { 6(Gal } \beta 1-4 G l c N A c \beta 1-3) \text { Gal } \beta 1- \\
\text { 4Glc }\end{array}$ & - & - \\
\hline $8 \mathrm{~L}$ & $\begin{array}{l}\text { Difucosyllacto-N- } \\
\text { neohexaose I } \\
\text { (DFLNnH I) }\end{array}$ & $\begin{array}{c}\text { Gal } \beta 1-4(\text { Fuc } \alpha 1-3) \text { GlcNAc } \beta 1- \\
\text { 6(Gal } \beta 1-4(\text { Fuc } \alpha 1- \\
\text { 3)GlcNAc } \beta 1-3) \text { Gal } \beta 1-4 G l c\end{array}$ & - & 2.97 \\
\hline $8 \mathrm{M}$ & $\begin{array}{l}\text { Difucosyllacto-N- } \\
\text { neohexaose II } \\
\text { (DFLNnH II) }\end{array}$ & $\begin{array}{c}\text { Fuc } \alpha 1-2 \text { Gal } \beta 1-4(\text { Fuc } \alpha 1- \\
\text { 3)GlcNAc } \beta 1-6(\text { Gal } \beta 1- \\
\text { 4GlcNAc } \beta b 1-3) \text { Gal } \beta 1-4 G l c\end{array}$ & - & 2.52 \\
\hline $8 \mathrm{~N}$ & $\begin{array}{l}\text { Monofucosyl(1-3)- } \\
\text { iso-lacto-N-octaose } \\
\text { (MFiLNO) }\end{array}$ & $\begin{array}{c}\text { Gal } \beta 1-3 \text { GlcNAc } \beta 1-3 \text { Gal } \beta 1- \\
\text { 4(Fuc } \alpha 1-3) \text { GlcNAc } \beta 1- \\
\text { 6(Gal } \beta 1-3 \text { GlcNAc } \beta 1-3) \text { Gal } \beta 1- \\
\text { 4Glc }\end{array}$ & - & - \\
\hline 80 & $\begin{array}{l}\text { Trifucosyl(1-2,1-2,1- } \\
\text { 3)-iso-lacto-N- } \\
\text { octaose (TFiLNO (1- } \\
2,1-2,1-3))\end{array}$ & $\begin{array}{c}\text { Fuc } \alpha 1-2 \text { Gal } \beta 1-3 \text { GlcNAc } \beta 1- \\
\text { 3Gal } \beta b 1-4(\text { Fuc } \alpha 1- \\
\text { 3)GlcNAc } \beta 1-6(\text { Gal } \beta 1- \\
\text { 3GlcNAc } \beta 1-3) \text { Gal } \beta 1-4 \text { Glc }\end{array}$ & - & 2.23 \\
\hline $8 \mathrm{P}$ & $\begin{array}{l}\text { Blood Group A } \\
\text { (tetra) }\end{array}$ & $\begin{array}{c}\text { GalNAc } \alpha 1-3(\text { Fuc } \alpha 1-2) \text { Gal } \beta 1- \\
\text { 4GalNAc }\end{array}$ & - & - \\
\hline $9 \mathrm{~A}$ & $\begin{array}{l}\text { Blood Group B } \\
\text { (penta) }\end{array}$ & $\begin{array}{c}\text { Gal } \alpha 1-3(\text { Fuc } \alpha 1-2) \mathrm{Gal} \beta 1- \\
\text { 4(Fuc } \alpha 1-3) \mathrm{Glc}\end{array}$ & - & - \\
\hline $9 \mathrm{~B}$ & $\begin{array}{l}\text { Monofucosyllacto-N- } \\
\text { hexaose I }\end{array}$ & $\begin{array}{c}\text { Gal } \beta 1-4 G l c N A c \beta 1-6(\text { Fuc } \alpha 1- \\
\text { 2Gal } \beta 1-3 \text { GlcNAc } \beta 1-3) \text { Gal } \beta 1- \\
\text { 4Glc }\end{array}$ & - & - \\
\hline \multicolumn{5}{|c|}{ Sialyl } \\
\hline 48 & $\alpha-\mathrm{Neu} 5 \mathrm{Ac}$ & Neu5Ac $\alpha-\operatorname{sp} 3$ & - & - \\
\hline 49 & $\alpha-N e u 5 A c B n$ & Neu5Aca-sp9 & - & - \\
\hline 52 & $\alpha-N e u 5 G c$ & Neu5Gca-sp3 & - & - \\
\hline 54 & 9-Nac- $\alpha-N e u 5 A c$ & 9-NAc-Neu5Ac $\alpha$-sp3 & 1.78 & - \\
\hline 169 & GM4 & Neu5Ac $\alpha 2-3$ Gal $\beta$-sp3 & - & - \\
\hline 170 & Sia6- $\beta$ Galactose & Neu5Ac $\alpha 2-6 \mathrm{Gal} \beta-\mathrm{sp} 3$ & - & - \\
\hline 171 & 3-SiaTn Antigen & Neu5Ac $\alpha 2-3$ GalNAc $\alpha$-sp3 & - & - \\
\hline 172 & SiaTn Antigen & Neu5Ac $\alpha 2-6$ GalNAc $\alpha$-sp3 & - & - \\
\hline 174 & NeuGc-Tn & Neu5Gc $\alpha 2-6$ GalNAc $\alpha$-sp3 & 3.13 & - \\
\hline
\end{tabular}




\begin{tabular}{|c|c|c|c|c|}
\hline 186 & (Sia)2 & Neu5Ac $\alpha 2-8$ Neu5Ac $\alpha 2-s p 3$ & - & - \\
\hline 205 & $\begin{array}{c}\text { Sia6- } \beta-N- \\
\text { acetylgalactosamine }\end{array}$ & Neu5Ac $\alpha 2-6$ GalNAc $\beta$-sp3 & - & - \\
\hline 206 & NeuGca3Galatacose & Neu5Gca2-3Gal-sp3 & - & - \\
\hline 289 & 6-SiaTF & $\begin{array}{l}\text { Gal } \alpha 1-3(\mathrm{Neu} 5 \mathrm{Ac} \alpha 2- \\
\text { 6)GalNAc } \alpha-\mathrm{sp} 3\end{array}$ & 1.50 & - \\
\hline 290 & $\mathrm{~A} 3 \mathrm{a}(\mathrm{Sia}) \mathrm{Tn}$ & $\begin{array}{l}\text { Gal } \beta 1-3(\text { Neu5Ac } \alpha 2- \\
\text { 6)GalNAc } \alpha \text {-sp3 }\end{array}$ & - & - \\
\hline 292 & 3-Sia-TF & $\begin{array}{l}\text { Neu5Aco2-3Gal } \beta 1- \\
\text { 3GalNAc } \alpha \text {-sp3 }\end{array}$ & - & - \\
\hline 293 & $\begin{array}{l}\text { 3'Sialyllactose } \\
\text { (3`SL) }\end{array}$ & Neu5Ac $\alpha 2-3$ Gal $\beta 1-4 G 1 c \beta-s p 3$ & - & - \\
\hline 294 & 3`Sialyllactose & Neu5Acc $2-3$ Gal $\beta 1-4 G 1 c \beta$-sp4 & - & - \\
\hline 295 & $\begin{array}{l}\text { 6. Sialyllactose } \\
\text { (6 SL) }\end{array}$ & Neu5Ac $\alpha 2-6$ Gal $\beta 1-4 G 1 c \beta-s p 2$ & 1.68 & - \\
\hline 298 & $\begin{array}{l}\text { 3'Sialyllactosamine } \\
\text { (3`SLN) }\end{array}$ & $\begin{array}{l}\text { Neu5Ac } \alpha 2-3 \text { Gal } \beta 1- \\
4 \text { GlcNAc } \beta \text {-sp3 }\end{array}$ & - & - \\
\hline 299 & 3`-SiaLeC & $\begin{array}{c}\text { Neu5Aco2-3Gal } \beta 1- \\
\text { 3GlcNAc } \beta \text {-sp3 }\end{array}$ & - & - \\
\hline 300 & $\begin{array}{l}\text { 6 Siallylactosamine } \\
\text { (6 SLN) }\end{array}$ & $\begin{array}{l}\text { Neu5Ac } \alpha 2-6 \text { Gal } \beta 1- \\
\text { 4GlcNAc } \beta \text {-sp3 }\end{array}$ & - & - \\
\hline 303 & $\begin{array}{l}\text { 3 Sialyllactosamine } \\
\text { (Gc) }\end{array}$ & $\begin{array}{l}\text { Neu5Gc } \alpha 2-3 \text { Gal } \beta 1- \\
4 \text { GlcNAc } \beta \text {-sp3 }\end{array}$ & - & - \\
\hline 304 & $\begin{array}{l}\text { 6 Sialyllactosamine } \\
(\mathrm{Gc})\end{array}$ & $\begin{array}{l}\text { Neu5Gco2-6Gal } \beta 1- \\
4 \text { GlcNAc } \beta \text {-sp3 }\end{array}$ & - & - \\
\hline 306 & 9NAc-6 SLN & $\begin{array}{l}\text { 9-NAc-Neu5Ac } \alpha 2-6 \text { Gal } \beta 1- \\
\text { 4GlcNAc } \beta \text {-sp3 }\end{array}$ & - & - \\
\hline 315 & 6-Su-3 'SLN & $\begin{array}{l}\text { Neu5Ac } \alpha 2-3 \text { Gal } \beta 1-4-(6-O- \\
\text { Su)GlcNAc } \beta \text {-sp3 }\end{array}$ & 1.39 & - \\
\hline 317 & 6-Su-3`SiaTF & $\begin{array}{c}\text { Neu5Aco2-3Gal } \beta 1-3-(6-\mathrm{O}- \\
\text { Su)GalNAc } \beta \text {-sp3 }\end{array}$ & - & - \\
\hline 318 & 6-Su-6 SLN & $\begin{array}{l}\text { Neu5Aca2-6Gal } \beta 1-4-(6-\mathrm{O}- \\
\text { Su)GlcNAc } \beta \text {-sp3 }\end{array}$ & - & - \\
\hline 319 & $6^{`}-\mathrm{Su}-3^{\prime} \mathrm{SLN}$ & $\begin{array}{c}\text { Neu5Ac } \alpha 2-3-(6-\mathrm{O}-\mathrm{Su}) \mathrm{Gal} \beta 1- \\
\text { 4GlcNAc } \beta-\mathrm{sp} 3\end{array}$ & - & - \\
\hline 321 & $(\mathrm{Sia}) 3$ & (Neu5Ac $\alpha 2-8) 3-s p 3$ & - & - \\
\hline 323 & 6`SiaLeC & $\begin{array}{l}\text { Neu5Aca2-6Galß1-3GlcNAc- } \\
\text { sp3 }\end{array}$ & - & - \\
\hline 324 & 6Su-6`-SiaLeC & $\begin{array}{l}\text { Neu5Ac } \alpha 2-6 \text { Gal } \beta 1-3(6-\mathrm{O}- \\
\text { Su)GlcNAc-sp3 }\end{array}$ & - & - \\
\hline 331 & 3'SiaLec (Gc) & $\begin{array}{c}\text { Neu5Gco2-3Gal } \beta 1- \\
\text { 3GlcNAc } \beta \text {-sp3 }\end{array}$ & 1.57 & - \\
\hline 421 & GM2 & $\begin{array}{c}\text { Neu5Ac } \alpha 2-3(\text { GalNAc } \beta 1- \\
\text { 4)Gal } \beta 1-4 \text { Glc } \beta \text {-sp2 }\end{array}$ & - & - \\
\hline 422 & 3`SLNb3A & $\begin{array}{c}\text { Neu5Ac } \alpha 2-3 \text { Gal } \beta 1- \\
\text { 4GlcNAc } \beta 1-3 \text { Gal } \beta \text {-sp3 }\end{array}$ & - & - \\
\hline 423 & SiaLeX & $\begin{array}{c}\text { Fuc } \alpha 1-3(\text { Neu5Ac } \alpha 2-3 \text { Gal } \beta 1- \\
\text { 4)GlcNAc } \beta \text {-sp3 }\end{array}$ & - & - \\
\hline
\end{tabular}




\begin{tabular}{|c|c|c|c|c|}
\hline 426 & SiaLeA & $\begin{array}{c}\text { Neu5Ac } \alpha 2-3 \text { Gal } \beta 1-3 \text { (Fuc } \alpha 1- \\
\text { 4)GlcNAc } \beta \text {-sp3 }\end{array}$ & - & - \\
\hline 428 & SiaLeX6Su & $\begin{array}{c}\text { Fuc } \alpha 1-3(\mathrm{Neu} 5 \mathrm{Ac} \alpha 2-3 \mathrm{Gal} \beta 1- \\
\text { 4)6-O-Su-GlcNAc } \beta \text {-sp3 }\end{array}$ & - & - \\
\hline 429 & SiaLeX6 ${ }^{` S u}$ & $\begin{array}{l}\text { Fuc } \alpha 1-3(\mathrm{Neu} 5 \mathrm{Ac} \alpha 2-3(6-\mathrm{O}- \\
\mathrm{Su}) \mathrm{Gal} \beta 1-4) \text { GlcNAc } \beta-\mathrm{sp} 3\end{array}$ & - & - \\
\hline 433 & $\mathrm{Sia}_{2}-\mathrm{TF}$ & $\begin{array}{c}\text { Neu5Ac } \alpha 2-3 \text { Gal } \beta 1- \\
\text { 3(Neu5Ac } \alpha 2-6) \text { GalNAc } \alpha-s p 3\end{array}$ & - & - \\
\hline 434 & GD3 & $\begin{array}{c}\text { Neu5Ac } \alpha 2-8 \text { Neu5Ac } \alpha 2- \\
3 \text { Gal } \beta 1-4 \text { Glc } \beta \text {-sp4 }\end{array}$ & - & - \\
\hline 527 & 3.SLN-LacNAc & $\begin{array}{c}\text { Neu5Ac } \alpha 2-3 \mathrm{Gal} \beta 1- \\
\text { 4GlcNAc } \beta 1-3 \mathrm{Gal} \beta 1- \\
\text { 4GlcNAc } \beta \text {-sp2 }\end{array}$ & - & - \\
\hline 528 & SiaLex-3Gal & $\begin{array}{c}\text { Fuc } \alpha 1-3(\text { Neu5Ac } \alpha 2-3 \mathrm{Gal} \beta 1- \\
\text { 4)GlcNAc } \beta 1-3 \mathrm{Gal} \beta \text {-sp3 }\end{array}$ & - & - \\
\hline 529 & $\mathrm{LSTb}$ & $\begin{array}{l}\text { Gal } \beta 1-3(\text { Neu5Ac } \alpha 2- \\
\text { 6)GlcNAc } \beta 1-3 \text { Gal } \beta 1-4 \text { Glc } \beta- \\
\text { sp4 }\end{array}$ & - & - \\
\hline 531 & GD2 & $\begin{array}{c}\text { GalNAc } \beta 1-4(\text { Neu5Ac } \alpha 2- \\
\text { 8Neu5Ac } \alpha 2-3) \text { Gal } \beta 1-4 G l c-s p 2\end{array}$ & - & - \\
\hline 532 & GT3 & $\begin{array}{c}\text { Neu5Ac } \alpha 2-8 \text { Neu5Ac } \alpha 2- \\
\text { 8Neu5Ac } \alpha 2-3 \text { Gal } \beta 1 \text {-4Glc-sp2 }\end{array}$ & - & - \\
\hline 533 & GT2 & $\begin{array}{c}\text { (Neu5Ac } \alpha 2-8) 2 \mathrm{Neu} 5 \mathrm{Ac} \alpha 2- \\
\text { 3(GalNAc } \beta 1-4) \text { Gal } \beta 1-4 \mathrm{Glc}- \\
\text { sp2 }\end{array}$ & - & - \\
\hline 534 & 6.SLN-LacNAc & $\begin{array}{c}\text { Neu5Ac } \alpha 2-3 \text { Gal } \beta 1- \\
\text { 4GlcNAc } \beta 1-3 \text { Gal } \beta 1- \\
\text { 4GlcNAc } \beta-\text { sp3 } \\
\end{array}$ & - & - \\
\hline 536 & LSTa & $\begin{array}{c}\text { Neu5Ac } 22-3 \mathrm{Gal} \beta 1- \\
\text { 3GlcNAc } \beta 1-3 \mathrm{Gal} \beta 1-4 \mathrm{Glc} \beta- \\
\text { sp4 }\end{array}$ & - & - \\
\hline 537 & LSTd & $\begin{array}{c}\text { Neu5Ac } \alpha 2-3 \text { Gal } \beta 1- \\
\text { 4GlcNAc } \beta 1-3 \text { Gal } \beta 1-4 \text { Glc } \beta- \\
\text { sp4 } \\
\end{array}$ & - & - \\
\hline 540 & MSMFLNnH & Lex1-6'(6'SLN1-3')Lac-sp4 & - & - \\
\hline 627 & $11-\mathrm{OS}$ & $\begin{array}{c}\text { (Sia2-6A-GN-M)2-3,6-M-GN- } \\
\text { GN } \beta-\text {-sp4 }\end{array}$ & - & - \\
\hline $10 \mathrm{~A}$ & SiaLewis A & $\begin{array}{c}\text { Neu5Ac } \alpha 2-3 \text { Gal } \beta 1-3(\text { Fuc } \alpha 1- \\
\text { 4)GlcNAc }\end{array}$ & - & - \\
\hline $10 \mathrm{~B}$ & SiaLewis $\mathrm{X}$ & $\begin{array}{c}\text { Neu5Ac } \alpha 2-3 \text { Gal } \beta 1-4(\text { Fuc } \alpha 1- \\
\text { 3)GlcNAc }\end{array}$ & - & - \\
\hline $10 \mathrm{D}$ & $\begin{array}{c}\text { Sialyllacto-N-tetraose } \\
\text { a }\end{array}$ & $\begin{array}{c}\text { Neu5Ac } \alpha 2-3 \mathrm{Gal} \beta 1- \\
\text { 3GlcNAc } \beta 1-3 \mathrm{Gal} \beta 1-4 \mathrm{Glc}\end{array}$ & - & 1.88 \\
\hline $10 \mathrm{D}$ & $\begin{array}{c}\text { Monosialyl, } \\
\text { monofucosyllacto- } N \text { - } \\
\text { neohexose } \\
\end{array}$ & $\begin{array}{c}\text { Gal } \beta 1-4(\text { Fuc } \alpha 1-3) \text { GlcNAc } \beta 1- \\
\text { 6(Neu5Ac } \alpha 2-6 \text { Gal } \beta 1- \\
\text { 4GlcNAc } \beta 1-3) \text { Galb1-4Glc }\end{array}$ & - & - \\
\hline $10 \mathrm{E}$ & Disialyl-TF & $\begin{array}{c}\text { Neu5Ac } \alpha 2-3 \text { Gal } \beta 1- \\
\text { 3(Neu5Ac } \alpha 2-6) \text { GalNAc }\end{array}$ & - & - \\
\hline
\end{tabular}




\begin{tabular}{|c|c|c|c|c|}
\hline $10 \mathrm{H}$ & $\begin{array}{l}\text { Sialyllacto-N- } \\
\text { fucopentaose VI }\end{array}$ & $\begin{array}{c}\text { Neu5Ac } \alpha 2-6 \text { Gal } \beta 1- \\
\text { 3GlcNAc } \beta 1-3 \text { Gal } \beta 1-4(\text { Fuc } \alpha 1- \\
\text { 3)Glc }\end{array}$ & - & 2.23 \\
\hline $10 \mathrm{I}$ & $\begin{array}{l}\text { Monosialyllacto-N- } \\
\text { hexaose }\end{array}$ & $\begin{array}{c}\text { Gal } \beta 1-3 \mathrm{GlcNAc} \beta 1- \\
\text { 3(Neu5Ac } \alpha 2-6 \mathrm{Gal} \beta 1- \\
\text { 4GlcNAc } \beta 1-6) \text { Gal } \beta 1-4 \mathrm{Glc}\end{array}$ & - & - \\
\hline $10 \mathrm{~J}$ & $\begin{array}{l}\text { Monosialyllacto-N- } \\
\text { neohexaose }\end{array}$ & $\begin{array}{c}\text { Neu5Ac } \alpha 2-6 \mathrm{Gal} \beta 1- \\
\text { 3GlcNAc } \beta 1-3(\mathrm{Gal} \beta 1- \\
\text { 4GlcNAc } \beta 1-6) \text { Gal } \beta 1-4 \mathrm{Glc}\end{array}$ & - & - \\
\hline $10 \mathrm{~K}$ & 3`-Sialyllactosamine & Neu5Ac $\alpha 2-3$ Gal $\beta 1-4 G l c N A c$ & - & 1.79 \\
\hline $10 \mathrm{~L}$ & 6-Sialyllactosamine & Neu5Ac $\alpha 2-6$ Gal $\beta 1-4 G l c N A c$ & - & - \\
\hline $10 \mathrm{M}$ & $\begin{array}{c}\text { LS-Tetrasaccharide a } \\
\text { (LSTa) }\end{array}$ & $\begin{array}{c}\text { Neu5Ac } \alpha 2-3 \mathrm{Gal} \beta 1- \\
\text { 3GlcNAc } \beta 1-3 \mathrm{Gal} \beta 1-4 \mathrm{Glc}\end{array}$ & - & - \\
\hline $10 \mathrm{~N}$ & $\begin{array}{c}\text { LS-Tetrasaccharide b } \\
(\mathrm{LST})\end{array}$ & $\begin{array}{c}\text { Gal } \beta 1-3(\text { Neu5Ac } \alpha 2- \\
\text { 6)GlcNAc } \beta 1-3 \text { Gal } \beta 1-4 G l c\end{array}$ & - & - \\
\hline $10 \mathrm{O}$ & $\begin{array}{c}\text { LS-Tetrasaccharide c } \\
(\mathrm{LSTc})\end{array}$ & $\begin{array}{c}\text { Neu5Ac } \alpha 2-6 \mathrm{Gal} \beta 1- \\
\text { 4GlcNAc } \beta 1-3 \mathrm{Gal} \beta 1-4 \mathrm{Glc}\end{array}$ & - & - \\
\hline $10 \mathrm{P}$ & $\begin{array}{l}\text { Disialyllacto- } N- \\
\text { tetraose }\end{array}$ & $\begin{array}{c}\text { Neu5Acc } 2-3 \text { Gal } \beta 1- \\
\text { 3(Neu5Ac } \alpha 2-6) \text { GlcNAc } \beta 1- \\
\text { 3Gal } \beta 1-4 \text { Glc }\end{array}$ & - & - \\
\hline $11 \mathrm{~A}$ & 3'-Sialyllactose & Neu5Ac $\alpha 2-3$ Gal $\beta 1-4 G l c$ & - & - \\
\hline $11 \mathrm{~B}$ & 6'-Sialyllactose & Neu5Ac $\alpha 2-6$ Gal $\beta 1-4 G l c$ & - & - \\
\hline $11 \mathrm{C}$ & Colominic acid & $\begin{array}{c}(\text { Neu5Ac } \alpha 2-8 \text { Neu5Ac)n } \\
(\mathrm{n}<50)\end{array}$ & - & - \\
\hline $11 \mathrm{D}$ & $\begin{array}{c}\text { Biantennary 2,6- } \\
\text { sialylated-N-glycan- } \\
\text { Asn }\end{array}$ & $\begin{array}{l}\text { Neu5Ac } \alpha 2-6 \text { Gal } \beta 1- \\
\text { 4GlcNAc } \beta 1-2 M a n \alpha 1- \\
\text { 6(Neu5Ac } \alpha 2-6 \text { Gal } \beta 1 \text { - } \\
\text { 4GlcNAc } \beta 1-2 M a n \alpha 1 \text { - } \\
\text { 6)Man } \beta 1-4 G l c N A c \beta 1- \\
\text { 4GlcNAc-Asn }\end{array}$ & - & - \\
\hline \multicolumn{5}{|c|}{ Terminal GalNAc } \\
\hline 4 & Tn Antigen & GalNAc $\alpha$-sp0 & 3.87 & - \\
\hline 5 & Tn Antigen & GalNAc $\alpha-s p 3$ & - & - \\
\hline 6 & $\beta$-GalNAc & GalNAc $\beta$-sp3 & - & - \\
\hline 38 & 3-O-Su- $\beta$-GalNac & 3-O-Su-GalNAco-sp3 & 1.04 & - \\
\hline 101 & Fs-2 & GalNAc $\alpha 1-3$ GalNAc $\beta$-sp3 & - & - \\
\hline 102 & Blood Group A (di) & GalNAc $\alpha 1-3$ Gal $\beta$-sp3 & - & - \\
\hline 103 & Core 5 & GalNAc $\alpha 1-3$ GalNAc $\alpha-s p 3$ & 2.39 & - \\
\hline 104 & $\mathrm{ANb} 3 \mathrm{~A}$ & GalNAc $\beta 1-3$ Gal $\beta$-sp3 & 2.18 & - \\
\hline 106 & LacdiNAc & GalNAc $\beta 1-4 G 1 c N A c \beta-s p 3$ & 3.83 & - \\
\hline 192 & 6-O-Su-LacdiNAc & $\begin{array}{l}\text { GalNAc } \beta 1-4(6-O- \\
\text { Su)GlcNAc } \beta-s p 3\end{array}$ & - & - \\
\hline 193 & 3`-O-Su-LacdiNAc & $\begin{array}{l}\text { 3-O-Su-GalNAc } \beta 1- \\
\text { 4GlcNAc } \beta \text {-sp3 }\end{array}$ & - & - \\
\hline 194 & 6-O-Su-LacdiNAc & $\begin{array}{c}\text { 6-O-Su-GalNAc } \beta 1- \\
\text { 4GlcNAc } \beta \text {-sp3 }\end{array}$ & - & - \\
\hline 195 & $\begin{array}{l}\text { 6-Su-3-O-Ac- } \\
\text { LacdiNAc }\end{array}$ & $\begin{array}{c}\text { 6-O-Su-GalNAc } \beta 1-4-(3-\mathrm{O}- \\
\mathrm{Su}) \text { GlcNAc } \beta \text {-sp3 }\end{array}$ & - & - \\
\hline
\end{tabular}




\begin{tabular}{|c|c|c|c|c|}
\hline 196 & $\begin{array}{l}3,3^{\prime}-\mathrm{O}-\mathrm{Su}_{2-} \\
\text { LacdiNAc }\end{array}$ & $\begin{array}{l}\text { 3-O-Su-GalNAc } \beta 1-4(3-\mathrm{O}-\mathrm{Su})- \\
\text { GlcNAc } \beta-\mathrm{sp} 3\end{array}$ & - & - \\
\hline 197 & $3,6-\mathrm{Su}_{2}$-LacdiNAc & $\begin{array}{l}\text { 3,6-O-Su2-GalNAc } \beta 1- \\
\text { 4GlcNAc } \beta \text {-sp3 }\end{array}$ & - & - \\
\hline 198 & $4^{\prime}, 6^{\prime}-\mathrm{Su}_{2}-\mathrm{LacdiNAc}$ & $\begin{array}{l}\text { 4,6-O-Su2-GalNAc } \beta 1- \\
\text { 4GlcNAc } \beta \text {-sp3 }\end{array}$ & 2.95 & - \\
\hline 199 & $\begin{array}{l}\text { 4,6 }{ }^{\prime}-\mathrm{Su}_{2}-3-\mathrm{O}-\mathrm{Ac}- \\
\text { LacdiNAc }\end{array}$ & $\begin{array}{c}\text { 4,6-O-Su2-GalNAc } \beta 1-4-(3-\mathrm{O}- \\
\text { Ac)GlcNAc } \beta \text {-sp3 }\end{array}$ & - & - \\
\hline 200 & 4-O-Su-LacdiNAc & $\begin{array}{l}\text { 4-O-Su-GalNAc } \beta 1- \\
\text { 4GlcNAc } \beta \text {-sp3 }\end{array}$ & - & - \\
\hline 202 & 6,6-O-Su-LacdiNAc & $\begin{array}{c}\text { 6-O-Su-GalNAc } \beta 1-4(6-\mathrm{O}- \\
\mathrm{Su}) \text { GlcNAc } \beta \text {-sp3 }\end{array}$ & - & - \\
\hline 204 & 4-O-Su-LacdiNAc & $\begin{array}{c}\text { 4-O-Su-GalNAc } \beta 1- \\
\text { 4GlcNAc } \beta \text {-sp2 }\end{array}$ & - & - \\
\hline 238 & GA2, GgOse3 & GalNAc $\beta 1-4$ Gal $\beta 1-4 \mathrm{Glc} \beta-\mathrm{sp} 3$ & - & - \\
\hline 389 & $\mathrm{~Gb} 4$ & $\begin{array}{c}\text { GalNac } \beta 1-3 \text { Gal } \alpha 1-4 \text { Gal } \beta 1- \\
4 \text { Glc } \beta-\operatorname{sp} 3\end{array}$ & - & - \\
\hline $1 \mathrm{~L}$ & Tn Antigen & GalNAc $\alpha 1-O-S e r$ & - & - \\
\hline $2 \mathrm{C}$ & $\begin{array}{c}\text { Terminal } \\
\text { disaccharide of } \\
\text { Globotriose } \\
\end{array}$ & GalNAc $\beta 1-3 \mathrm{Gal}$ & - & - \\
\hline $2 \mathrm{E}$ & $\begin{array}{c}\text { a-D- } N \text { - } \\
\text { acetylgalactosamine } \\
1-3 \mathrm{Gal} \beta 1-4 \mathrm{Glc}\end{array}$ & GalNAc $\alpha 1-3 \mathrm{Gal} \beta 1-4 \mathrm{Glc}$ & - & - \\
\hline $2 \mathrm{~F}$ & $\begin{array}{c}\text { a-D-N- } \\
\text { acetylgalactosamine } \\
1-3 \mathrm{Gal} \beta 1-4 \mathrm{Glc}\end{array}$ & GalNAc $\alpha 1-3$ Gal $\beta 1-4 \mathrm{Glc}$ & - & - \\
\hline \multicolumn{5}{|c|}{ Terminal Glc } \\
\hline 7 & $\alpha-G l c$ & Glc $\alpha-\operatorname{sp} 3$ & - & - \\
\hline 9 & $\beta-G l c$ & Glc $\beta$-sp3 & - & - \\
\hline 46 & $\beta$-Glc6P & 6-H2PO3Glc $\beta$-sp4 & - & - \\
\hline 110 & Maltose & Glc $\alpha 1-4 G 1 c \beta-s p 3$ & - & - \\
\hline 111 & Cellobiose & Glc $\beta 1-4$ Glc $\beta$-sp4 & - & - \\
\hline 112 & Gentiobiose & Glc $\beta 1-6$ Glc $\beta-\mathrm{sp} 4$ & - & - \\
\hline 240 & Maltotriose & $(\mathrm{Glc} \alpha 1-4) 3 \beta-\mathrm{sp} 4$ & - & - \\
\hline 241 & Isomaltotriose & $(\mathrm{Glc} \alpha 1-6) 3 \beta-\mathrm{sp} 4$ & 1.78 & - \\
\hline 390 & Maltotetraose & $(\mathrm{Glc} \alpha 1-4) 4 \beta-\mathrm{sp} 4$ & - & - \\
\hline 391 & Isomaltotetraose & $($ Glc $\alpha 1-6) 4 \beta-s p 4$ & - & - \\
\hline 492 & Isomaltopentaose & $($ Glc $\alpha 1-6) 5 \beta-\mathrm{sp} 4$ & - & - \\
\hline 502 & Maltohexaose & $($ Glc $\alpha 1-6) 6 \beta-s p 4$ & - & - \\
\hline \multicolumn{5}{|c|}{ Other } \\
\hline 14 & $\beta-\mathrm{GlcN}(\mathrm{Gc})$ & GlcN(Gc) $\beta$-sp4 & 2.09 & 1.07 \\
\hline 15 & Aminoglucitol & $\mathrm{HOCH} 2(\mathrm{HOCH}) 4 \mathrm{CH} 2 \mathrm{NH} 2$ & 2.48 & - \\
\hline 20 & L- $\alpha-$ Rha & Rha $\alpha-$ sp3 & - & - \\
\hline 44 & $\alpha$-Glucoronic acid & GlcA $\alpha$-sp3 & - & - \\
\hline 45 & $\beta$-Glucoronic acid & GlcA $\beta$-sp3 & - & - \\
\hline 164 & GUb3GN & GlcA $\beta 1-3$ GlcNAc $\beta$-sp3 & - & - \\
\hline 165 & GUb3A & GlcA $\beta 1-3 \mathrm{Gal} \beta-\mathrm{sp} 3$ & - & - \\
\hline 166 & GUb6A & GlcA $\beta 1-6 \mathrm{Gal} \beta-\mathrm{sp} 3$ & - & - \\
\hline
\end{tabular}




\begin{tabular}{|c|c|c|c|c|}
\hline \multicolumn{5}{|c|}{ Glycosaminoglycans (GAGs) and Glucans } \\
\hline 625 & HyalU-ol & $\begin{array}{l}(\text { GlcA } \beta 1-4 G 1 c N A c \beta 1-3) 8- \\
\text { NH2-ol }\end{array}$ & - & - \\
\hline $12 \mathrm{~A}$ & $\begin{array}{c}\mathrm{C}_{24} \mathrm{H}_{36} \mathrm{O}_{25} \mathrm{~S}_{2} \mathrm{Na}_{2} \\
\text { (Mixed anomers. } \\
\text { Tetrasaccharide of } \\
\text { regular } \kappa \text { - } \\
\text { carrageenan) }\end{array}$ & $\begin{array}{l}\text { Neocarratetraose-41, 3-di-O- } \\
\text { sulphate }(\mathrm{Na}+)\end{array}$ & - & - \\
\hline $12 \mathrm{~B}$ & $\begin{array}{c}\mathrm{C}_{24} \mathrm{H}_{37} \mathrm{O}_{22} \mathrm{SNa} \\
\text { (Mixed anomers. } \\
\text { Derived from C1003 } \\
\text { by removal of the } \\
\text { non-reducing } \\
\text { terminal 4-sulphate) }\end{array}$ & $\begin{array}{l}\text { Neocarratetraose-41-O- } \\
\text { sulphate }(\mathrm{Na}+)\end{array}$ & - & - \\
\hline $12 \mathrm{C}$ & $\begin{array}{c}\mathrm{C}_{36} \mathrm{H}_{52} \mathrm{O}_{40} \mathrm{~S}_{4} \mathrm{Na}_{4} \\
\text { (Mixed anomers. A } \\
\text { hybrid sequence } \\
\text { comprising } \\
\text { carrageenan } \\
\text { disaccharides in the } \\
\text { order k-i-k, derived } \\
\text { from the carrageenan } \\
\text { from Chondrus } \\
\text { crispus) }\end{array}$ & $\begin{array}{l}\text { Neocarrahexaose-24,41, 3, 5- } \\
\text { tetra-O-sulphate }(\mathrm{Na}+)\end{array}$ & - & - \\
\hline $12 \mathrm{D}$ & $\begin{array}{c}\mathrm{C}_{36} \mathrm{H}_{53} \mathrm{O}_{37} \mathrm{~S}_{3} \mathrm{Na}_{3} \\
\text { (Mixed anomers. } \\
\text { Hexasaccharide of } \\
\text { regular } \kappa- \\
\text { carrageenan) }\end{array}$ & $\begin{array}{c}\text { Neocarrahexaose-41, 3, 5-tri- } \\
\text { O-sulphate }(\mathrm{Na}+)\end{array}$ & - & - \\
\hline $12 \mathrm{E}$ & $\begin{array}{c}\mathrm{C}_{48} \mathrm{H}_{70} \mathrm{O}_{49} \mathrm{~S}_{4} \mathrm{Na}_{4} \\
\text { (Mixed anomers. } \\
\text { Octasaccharide of } \\
\text { regular } \kappa- \\
\text { carrageenan) } \\
\end{array}$ & $\begin{array}{l}\text { Neocarraoctaose- } 41,3,5,7- \\
\text { tetra-O-sulphate }(\mathrm{Na}+)\end{array}$ & - & - \\
\hline $12 \mathrm{~F}$ & $\begin{array}{c}\mathrm{C}_{60} \mathrm{H}_{87} \mathrm{O}_{61} \mathrm{~S}_{5} \mathrm{Na}_{5} \\
\text { (Mixed anomers. } \\
\text { Decasaccharide of } \\
\text { regular } \kappa- \\
\text { carrageenan) }\end{array}$ & $\begin{array}{c}\text { Neocarradecaose-41, } 3,5,7,9- \\
\text { penta-O-sulphate }(\mathrm{Na}+)\end{array}$ & - & - \\
\hline $12 \mathrm{G}$ & $\begin{array}{c}\mathrm{C}_{12} \mathrm{H}_{15} \mathrm{NO}_{19} \mathrm{~S}_{3} \mathrm{Na}_{4} \\
\text { (Predominant } \\
\text { disaccharide } \\
\text { produced from } \\
\text { heparin by heparinase } \\
\text { I and II) }\end{array}$ & $\Delta \mathrm{UA}-2 \mathrm{~S}-\mathrm{GlcNS}-6 \mathrm{~S} \mathrm{Na} 4(\mathrm{I}-\mathrm{S})$ & - & - \\
\hline $12 \mathrm{H}$ & $\begin{array}{c}\mathrm{C}_{12} \mathrm{H}_{16} \mathrm{NO}_{16} \mathrm{~S}_{2} \mathrm{Na}_{3} \\
\text { (Produced from } \\
\text { heparinase II } \\
\text { digestion of heparin } \\
\text { and heparin sulphate) }\end{array}$ & $\Delta \mathrm{UA} \rightarrow$ GlucNS-6S Na3 (II-S) & - & - \\
\hline
\end{tabular}




\begin{tabular}{|c|c|c|c|c|}
\hline $12 \mathrm{I}$ & $\begin{array}{c}\mathrm{C}_{12} \mathrm{H}_{16} \mathrm{NO}_{16} \mathrm{~S}_{2} \mathrm{Na}_{3} \\
\text { (Produced from } \\
\text { heparin by digestion } \\
\text { with heparinase I and } \\
\text { II) }\end{array}$ & $\Delta \mathrm{UA} \rightarrow 2 \mathrm{~S}-\mathrm{GlcNS} \mathrm{Na}_{3}(\mathrm{III}-\mathrm{S})$ & - & - \\
\hline $12 \mathrm{~J}$ & $\begin{array}{c}\mathrm{C}_{14} \mathrm{H}_{18} \mathrm{NO}_{17} \mathrm{~S}_{2} \mathrm{Na}_{3} \\
\text { (Minor component } \\
\text { produced from } \\
\text { heparin by heparinase } \\
\text { II) }\end{array}$ & $\begin{array}{c}\Delta \mathrm{UA} \rightarrow \underset{(\mathrm{I}-\mathrm{A})}{2 \mathrm{~S}-\mathrm{GlcNAc}-6 \mathrm{~S} \mathrm{Na}} \\
\text { Na3 }\end{array}$ & - & - \\
\hline $12 \mathrm{~K}$ & $\begin{array}{l}\mathrm{C}_{14} \mathrm{H}_{19} \mathrm{NO}_{14} \mathrm{SNa}_{2} \\
\text { (Product of the action } \\
\text { of heparinases II and } \\
\text { III on heparin and } \\
\text { heparan sulphate) }\end{array}$ & $\begin{array}{c}\Delta \mathrm{UA} \rightarrow \text { GlcNAc-6S Na}(\mathrm{II}- \\
\mathrm{A})\end{array}$ & - & - \\
\hline $12 \mathrm{~L}$ & $\begin{array}{c}\mathrm{C}_{14} \mathrm{H}_{19} \mathrm{NO}_{14} \mathrm{SNa}_{2} \\
\text { (Minor product of the } \\
\text { action of heparinase } \\
\text { II on heparin) }\end{array}$ & $\Delta \mathrm{UA} \rightarrow \underset{\mathrm{A} \text { ) }}{2 \mathrm{~S}-\mathrm{GlcNAc} \mathrm{Na}_{2} \text { (III- }}$ & - & - \\
\hline $12 \mathrm{M}$ & $\begin{array}{l}\mathrm{C}_{14} \mathrm{H}_{20} \mathrm{NO}_{11} \mathrm{Na} \\
\text { (Produced from } \\
\text { heparin sulphate by } \\
\text { digestion With } \\
\text { heparinase III) }\end{array}$ & $\Delta \mathrm{UA} \rightarrow \mathrm{GlcNAc} \mathrm{Na}(\mathrm{IV}-\mathrm{A})$ & - & - \\
\hline $12 \mathrm{~N}$ & $\begin{array}{l}\mathrm{C}_{14} \mathrm{H}_{19} \mathrm{NO}_{14} \mathrm{SNa}_{2} \\
\text { (Produced from } \\
\text { various chondroitin } \\
\text { sulphates By the } \\
\text { action of } \\
\text { chondroitinases } \\
\mathrm{ABC}, \mathrm{B} \text { and } \mathrm{AC}-1 \text { ) }\end{array}$ & $\Delta \mathrm{UA} \rightarrow \underset{\mathrm{Di}-4 \mathrm{~S})}{\text { GalNAc- } 4 \mathrm{~S} \mathrm{Na}_{2}(\Delta}$ & - & - \\
\hline $12 \mathrm{O}$ & $\begin{array}{l}\mathrm{C}_{14} \mathrm{H}_{19} \mathrm{NO}_{14} \mathrm{SNa}_{2} \\
\text { (Produced from } \\
\text { various chondroitin } \\
\text { sulphates By the } \\
\text { action of } \\
\text { chondroitinases } \\
\text { ABC, AC-1 and C) }\end{array}$ & $\Delta \mathrm{UA} \rightarrow \underset{\text { Di-6S })}{\text { GalNAc-6S Na2 }(\Delta}$ & - & - \\
\hline $12 \mathrm{P}$ & $\begin{array}{c}\mathrm{C}_{14} \mathrm{H}_{18} \mathrm{NO}_{17} \mathrm{~S}_{2} \mathrm{Na}_{3} \\
\text { (Produced from } \\
\text { various chondroitin } \\
\text { sulphates By the } \\
\text { action of } \\
\text { chondroitinases } \\
\mathrm{ABC}, \mathrm{B} \text { and } \mathrm{AC}-1 \text { ) }\end{array}$ & $\Delta \mathrm{UA} \rightarrow \underset{\text { GalNAc- } 4 \mathrm{~S}, 6 \mathrm{~S} \mathrm{Na} \mathrm{Na}_{3}(\Delta}{\text { Di-disE })}$ & - & - \\
\hline $13 \mathrm{~A}$ & $\begin{array}{c}\mathrm{C}_{14} \mathrm{H}_{18} \mathrm{NO}_{17} \mathrm{~S}_{2} \mathrm{Na}_{3} \\
\text { (Produced from } \\
\text { various chondroitin } \\
\text { sulphates by action of } \\
\text { chondroitinase } \mathrm{ABC}\end{array}$ & $\begin{aligned} \Delta \mathrm{UA} \rightarrow & 2 \mathrm{~S}-\text { GalNAc-4S Na2 } \\
& (\Delta \text { Di-disB })\end{aligned}$ & - & - \\
\hline
\end{tabular}




\begin{tabular}{|c|c|c|c|c|}
\hline & $\begin{array}{c}\text { and/or B. Most } \\
\text { typically from } \\
\text { chondroitin sulphate } \\
\text { B (dermatan } \\
\text { sulphate)) }\end{array}$ & & & \\
\hline 13B & $\begin{array}{c}\mathrm{C}_{14} \mathrm{H}_{18} \mathrm{NO}_{17} \mathrm{~S}_{2} \mathrm{Na}_{3} \\
\text { (Produced from } \\
\text { various chondroitin } \\
\text { sulphates by the } \\
\text { action of } \\
\text { chondroitinase } \mathrm{ABC} \text { ) }\end{array}$ & $\begin{array}{c}\Delta \mathrm{UA} \rightarrow \underset{\text { Di-disD })}{2 \mathrm{~S}-\text { GalNAc-6S Na3 }}(\Delta \\
\text { Dis }\end{array}$ & - & - \\
\hline $13 \mathrm{C}$ & $\begin{array}{c}\mathrm{C}_{14} \mathrm{H}_{17} \mathrm{NO}_{20} \mathrm{~S}_{3} \mathrm{Na}_{4} \\
\text { (Produced as a minor } \\
\text { component by the } \\
\text { action of } \\
\text { chondroitinase } \mathrm{ABC} \\
\text { on various } \\
\text { chondroitin } \\
\text { sulphates, } \\
\text { particularly B) }\end{array}$ & $\begin{array}{c}\Delta \mathrm{UA} \rightarrow \underset{\mathrm{Na}}{\rightarrow} \text { 2S-GalNAc-4S-6S } \\
\mathrm{Na}_{4}(\Delta \text { Di-tisS })\end{array}$ & - & - \\
\hline $13 \mathrm{D}$ & $\begin{array}{l}\mathrm{C}_{14} \mathrm{H}_{19} \mathrm{NO}_{14} \mathrm{SNa} 2 \\
\text { (Produced as a minor } \\
\text { component from } \\
\text { various chondroitin } \\
\text { sulphates by the } \\
\text { action of } \\
\text { chondroitinase } \mathrm{ABC} \text { ) }\end{array}$ & $\begin{aligned} \Delta \mathrm{UA} \rightarrow & 2 \mathrm{~S}-\text { GalNAc-6S Na2 } \\
& (\Delta \mathrm{Di}-\mathrm{UA} 2 \mathrm{~S})\end{aligned}$ & - & - \\
\hline $13 \mathrm{E}$ & $\begin{array}{l}\mathrm{C}_{14} \mathrm{H}_{20} \mathrm{NO}_{11} \mathrm{Na} \text { (The } \\
\text { only unsaturated } \\
\text { disaccharide } \\
\text { produced from } \\
\text { hyaluronic acid by } \\
\text { the action of } \\
\text { chondroitinase ABC } \\
\text { or AC-1) }\end{array}$ & $\Delta \mathrm{UA} \rightarrow \underset{\mathrm{HA})}{\mathrm{GlcNAc}} \mathrm{Na}(\Delta \mathrm{Di}-$ & - & - \\
\hline $13 \mathrm{~F}$ & $\begin{array}{l}\text { Hyaluronic acid } \\
\text { (hyaluronan) }\end{array}$ & $\begin{array}{c}(\text { GlcA } \beta 1-3 \text { GlcNAc } \beta 1-4) n \\
(n=4)\end{array}$ & - & - \\
\hline $13 \mathrm{G}$ & $\begin{array}{l}\text { Hyaluronic acid } \\
\text { (hyaluronan) }\end{array}$ & $\begin{array}{c}(\text { GlcA } \beta 1-3 \text { GlcNAc } \beta 1-4) n \\
(n=8)\end{array}$ & - & - \\
\hline $13 \mathrm{H}$ & $\begin{array}{l}\text { Hyaluronic acid } \\
\text { (hyaluronan) }\end{array}$ & $\begin{array}{c}(\text { GlcA } \beta 1-3 \text { GlcNAc } \beta 1-4) n \\
(n=10)\end{array}$ & - & - \\
\hline $13 \mathrm{I}$ & $\begin{array}{l}\text { Hyaluronic acid } \\
\text { (hyaluronan) }\end{array}$ & $\begin{array}{c}(\text { GlcA } \beta 1-3 \text { GlcNAc } \beta 1-4) n \\
(n=12)\end{array}$ & - & - \\
\hline $13 \mathrm{~J}$ & Heparin & $\begin{array}{c}(\mathrm{GlcA} / \mathrm{IdoA} \pm 2 \mathrm{~S} \alpha / \beta 1- \\
4 \mathrm{GlcNAc} \pm \mathrm{NS} / 3 \mathrm{~S} / 6 \mathrm{~S} \alpha 1-4) \mathrm{n} \\
(\mathrm{n}=200)\end{array}$ & - & - \\
\hline $13 \mathrm{~K}$ & Chondroitin sulfate & $\begin{array}{c}\text { (GlcA/IdoA } \beta 1- \\
3( \pm 4 / 6 \mathrm{~S}) \text { GalNAc } \beta 1-4) n \\
(\mathrm{n}<250)\end{array}$ & - & - \\
\hline
\end{tabular}




\begin{tabular}{|c|c|c|c|c|}
\hline $13 \mathrm{~L}$ & Dermatan sulfate & $\begin{array}{c}(( \pm 2 \mathrm{~S}) \text { GlcA/IdoA } \alpha / \mathrm{b} 1- \\
3( \pm 4 \mathrm{~S}) \text { GalNAc } \beta 1-4) n(n<250)\end{array}$ & - & - \\
\hline $13 \mathrm{M}$ & Chondroitin 6-sulfate & $\begin{array}{c}(\text { GlcA/IdoA } \beta 1- \\
3( \pm 6 \mathrm{~S}) \text { GalNAc } \beta 1-4) n(n<250)\end{array}$ & - & - \\
\hline $13 \mathrm{~N}$ & $\begin{array}{l}\text { Hyaluronic acid } \\
\text { (hyaluronan) }\end{array}$ & $\begin{array}{c}(\text { GlcA } \beta 1-3 G l c N A c \beta 1-4) n \\
(n=4)\end{array}$ & - & - \\
\hline 130 & $\begin{array}{l}\text { Hyaluronic acid } \\
\text { (hyaluronan) }\end{array}$ & $\begin{array}{c}(\text { GlcA } \beta 1-3 G l c N A c \beta 1-4) n \\
(n=6)\end{array}$ & - & - \\
\hline $13 \mathrm{P}$ & $\begin{array}{l}\text { Hyaluronic acid } \\
\text { (hyaluronan) }\end{array}$ & $\begin{array}{c}(\text { GlcA } \beta 1-3 G l c N A c \beta 1-4) n \\
(n=8)\end{array}$ & - & - \\
\hline $14 \mathrm{~A}$ & $\begin{array}{l}\text { Hyaluronic acid } \\
\text { (hyaluronan) }\end{array}$ & $\begin{array}{l}(\text { GlcA } \beta 1-3 G l c N A c \beta 1-4) n \\
(n=10)\end{array}$ & - & - \\
\hline 14B & $\begin{array}{l}\text { Hyaluronic acid } \\
\text { (hyaluronan) }\end{array}$ & $\begin{array}{c}(\text { GlcA } \beta 1-3 G l c N A c \beta 1-4) n \\
(n=12)\end{array}$ & - & - \\
\hline $14 \mathrm{C}$ & $\begin{array}{l}\text { Hyaluronic acid } \\
\text { (hyaluronan) }\end{array}$ & $\begin{array}{c}(\text { GlcA } \beta 1-3 G l c N A c \beta 1-4) n \\
(n=14)\end{array}$ & - & - \\
\hline $14 \mathrm{D}$ & $\begin{array}{l}\text { Hyaluronic acid } \\
\text { (hyaluronan) }\end{array}$ & $\begin{array}{c}(\text { GlcA } \beta 1-3 G l c N A c \beta 1-4) n \\
(n=16)\end{array}$ & - & - \\
\hline $14 \mathrm{E}$ & $\begin{array}{c}\text { Hyaluronic acid } \\
\text { (hyaluronan) } \\
30,000 \mathrm{da}\end{array}$ & $($ GlcA $\beta 1-3$ GlcNAc $\beta 1-4) n$ & - & - \\
\hline $14 \mathrm{~F}$ & $\begin{array}{c}\text { Hyaluronic acid } \\
\text { (hyaluronan) } 107,000 \\
\text { da }\end{array}$ & $($ GlcA $\beta 1-3$ GlcNAc $\beta 1-4) n$ & - & 2.67 \\
\hline $14 \mathrm{G}$ & $\begin{array}{c}\text { Hyaluronic acid } \\
\text { (hyaluronan) } \\
190,000 \mathrm{da} \\
\end{array}$ & $($ GlcA $\beta 1-3$ GlcNAc $\beta 1-4) n$ & - & - \\
\hline $14 \mathrm{H}$ & $\begin{array}{l}\text { Hyaluronic acid } \\
\text { (hyaluronan) } \\
220,000 \mathrm{da} \\
\end{array}$ & $($ GlcA $\beta 1-3$ GlcNAc $\beta 1-4) n$ & - & - \\
\hline $14 \mathrm{I}$ & $\begin{array}{l}\text { Hyaluronic acid } \\
\text { (hyaluronan) } \\
1,600,000 \mathrm{da}\end{array}$ & $($ GlcA $\beta 1-3$ GlcNAc $\beta 1-4) n$ & - & 2.20 \\
\hline $14 \mathrm{~J}$ & Heparan Sulfate & $\begin{array}{c}(\mathrm{GlcA} / \mathrm{IdoA} \pm 2 \mathrm{~S} \alpha / \beta 1- \\
4 \mathrm{GlcNAc} \pm \mathrm{NS} / 3 \mathrm{~S} / 6 \mathrm{~S} \alpha 1-4) \mathrm{n} \\
(\mathrm{n}=200)\end{array}$ & - & 1.35 \\
\hline $14 \mathrm{~K}$ & $\beta 1-3$ Glucan & (Glc $\beta 1-3 G l c) n$ & - & 1.75 \\
\hline $14 \mathrm{~L}$ & $\begin{array}{l}\text { Chondroitin } \\
\text { disaccharide } \Delta \text { di-OS, } \\
\text { sodium salt }\end{array}$ & $\begin{array}{c}\mathrm{C}_{14} \mathrm{H}_{20} \mathrm{NNaO}_{11} \text { (produced from } \\
\text { various chondriotin sulfates by } \\
\text { the action of chondriotinases } \\
\mathrm{ABC}, \mathrm{AC}-1 \text { and } \mathrm{C} \text { ) }\end{array}$ & - & - \\
\hline $14 \mathrm{M}$ & $\begin{array}{l}\text { Heparin unsaturated } \\
\text { disaccharide I-H, the } \\
\text { major component } \\
\text { from the action of } \\
\text { heparinase II on de- } \\
\mathrm{N} \text {-sulfated heparin }\end{array}$ & $\Delta \mathrm{UA} \rightarrow 2 \mathrm{~S}-\mathrm{GlcN}-6 \mathrm{~S}$ & - & - \\
\hline $14 \mathrm{~N}$ & $\begin{array}{l}\text { Heparin unsaturated } \\
\text { disaccharide II-H, the }\end{array}$ & $\Delta \mathrm{UA} \rightarrow \mathrm{GlcN}-6 \mathrm{~S}$ & - & - \\
\hline
\end{tabular}




\begin{tabular}{|c|c|c|c|c|}
\hline & $\begin{array}{c}\text { major component } \\
\text { from the action of } \\
\text { heparinase II on de- } \\
\text { N-sulfated heparin }\end{array}$ & $\begin{array}{l}\text { Heparin unsaturated } \\
\text { disaccharide III-H, } \\
\text { the major component } \\
\text { from the action of } \\
\text { heparinase II on de- } \\
\text { N-sulfated heparin }\end{array}$ \\
\hline $14 \mathrm{O}$ & $\begin{array}{c}\text { Heparin unsaturated } \\
\text { disaccharide iV-H is } \\
\text { obtained from the } \\
\text { action of heparinases } \\
\text { on heparin }\end{array}$ & $\Delta \mathrm{UA} \rightarrow 2 \mathrm{~S}-\mathrm{GlcN}$ & - & - \\
\hline & $\Delta \mathrm{UA} \rightarrow \mathrm{GlcN}$ & - & - \\
\hline
\end{tabular}

Fold-change values for each glycan are calculated by the mean relative fluorescence units of four replicate spots divided by the mean background, defined as the average background of negative control spots plus 3 standard deviations. - indicates a fold-change value that was below 1 . 
Table S2. Glycan Array Document Based on MIRAGE Guidelines'.

\begin{tabular}{|l|l|}
\hline Classification & \multicolumn{1}{c|}{ Guidelines } \\
\hline 1. Sample: Glycan Binding Sample \\
\hline $\begin{array}{l}\text { Sample names: } \\
\text { Plasmodium sporozoites } \\
\text { Origin: Plasmodium falciparum sporozoites isolated from mosquito salivary glands. } \\
\text { Sample }\end{array}$ & $\begin{array}{l}\text { Origin: Plasmodium yoelii sporozoites isolated from mosquito salivary glands. } \\
\text { Method of preparation: }\end{array}$ \\
\hline $\begin{array}{l}\text { The isolation of sporozoites are explained in the Methods section. } \\
\text { modifications }\end{array}$ & Samples are labelled with CFDA-SE, see Methods section. \\
\hline Assay protocol & Please see Methods section. \\
\hline
\end{tabular}

2. Glycan Library

\begin{tabular}{|l|l|}
\hline $\begin{array}{l}\text { Glycan } \\
\text { description for } \\
\text { defined } \\
\text { glycans }\end{array}$ & $\begin{array}{l}\text { Glycans bound in this study are listed in Supplementary Table 1 and the full list of } \\
\text { glycans is a published library }\end{array}$ \\
\hline $\begin{array}{l}\text { Glycan } \\
\text { description for } \\
\text { undefined } \\
\text { glycans }\end{array}$ & N/A. \\
\hline $\begin{array}{l}\text { Glycan } \\
\text { modifications }\end{array}$ & $\begin{array}{l}\text { Glycans were prepared in one of two ways for printing: } \\
\text { 1. Glycans (with IDs in number/letter format; e.g. 1A, 4C, 7K) were sourced } \\
\text { commercially from Dextra Laboratories, Elicityl and Carbosythn and were made into } \\
\text { glycoamines using the protocol published in Day et al. 2009 }\end{array}$ \\
$\begin{array}{l}\text { 2. Glycans (with IDs in number only format) were obtained from Prof Nicolai Bovin } \\
\text { and were modified with spacers (sp0, sp2, sp3, sp4) as per. The library of these } \\
\text { glycans was first published in }\end{array}$ \\
\hline
\end{tabular}

\begin{tabular}{|l|l|}
\hline \multicolumn{2}{|l|}{ 3. Printing Surface; e.g., Microarray Slide } \\
\hline $\begin{array}{l}\text { Description of } \\
\text { surface }\end{array}$ & Epoxy activated glass microarray slides. \\
\hline Manufacturer & ArrayIt SuperEpoxy 3 (SME3). \\
\hline $\begin{array}{l}\text { Custom } \\
\text { preparation of } \\
\text { surface }\end{array}$ & N/A. \\
\hline
\end{tabular}




\begin{tabular}{|c|c|}
\hline $\begin{array}{l}\text { Non-covalent } \\
\text { Immobilisation }\end{array}$ & N/A. \\
\hline \multicolumn{2}{|c|}{ 4. Arrayer (Printer) } \\
\hline $\begin{array}{l}\text { Description of } \\
\text { Arrayer }\end{array}$ & SpotBot ${ }^{\circledR}$ Extreme Protein Microarray Spotter (ArrayIt, California, USA). \\
\hline $\begin{array}{l}\text { Dispensing } \\
\text { mechanism }\end{array}$ & $\begin{array}{l}\text { Contact printing using } 946 \mathrm{NS} 4 \text { pins with a } 6 \text { pin in a } 3 \text { columns } \mathrm{x} 2 \text { rows } \\
\text { configuration. }\end{array}$ \\
\hline $\begin{array}{l}\text { Glycan } \\
\text { deposition }\end{array}$ & $\begin{array}{l}\text { Approximately } 1.8 \mathrm{nl} \text { per spot is printed according to manufactures guidelines. } \\
\text { Glycan were at } 500 \mu \mathrm{M} \text { in 50:50 DMF:DMSO. }\end{array}$ \\
\hline $\begin{array}{l}\text { Printing } \\
\text { conditions }\end{array}$ & $\begin{array}{l}\text { Array were printed with dehumidification at a maximum humidity of } 60 \% \text { relative } \\
\text { humidity (Standard laboratory starting humidity of } 75-90 \% \text { ) at } 22^{\circ} \mathrm{C} \text {. Glycans were left } \\
\text { to react with the slide for at least } 8 \text { hours after the print was completed. }\end{array}$ \\
\hline \multicolumn{2}{|c|}{ 5. Glycan Microarray with "Map" } \\
\hline Array layout & $\begin{array}{l}\text { The array consists of a single array of glycans split between } 6 \text { pins ( } 3 \text { columns x } 2 \text { rows) } \\
\text { with } 4500 \mu \mathrm{m} \text { row and column spacing. Each pin printed a } 20 \text { columns x } 16 \text { rows with } \\
200 \mu \mathrm{m} \text { spot spacing (centre to centre) with a minimum spot size of } 100 \mu \mathrm{m} \text {. Each sample } \\
\text { is printed in quadruplicate with each of the } 6 \text { print areas including at least three negative } \\
\text { control samples (print solution only) and two positive control samples consisting of one } \\
\text { sample of fluoroscienamine and one sample of a mixture of rabbit anti-mouse antibody } \\
\text { labeled with Alexa } 555 \text { and Alexa } 647 \text {. Positive controls provide proof of successful } \\
\text { immobilization of the amine reagents and provides for orientation for analysis. The } \\
\text { antibodies also can provide controls for secondary antibodies used in experiments (if } \\
\text { applicable). }\end{array}$ \\
\hline $\begin{array}{l}\text { Glycan } \\
\text { identification } \\
\text { and quality } \\
\text { control }\end{array}$ & $\begin{array}{l}\text { Arrays are quality controlled by a range of measures. } 1 \text {. Each printed array is post print } \\
\text { scanned to confirm deposition of the glycans on the array surface prior to neutralization } \\
\text { of the remaining slide surface. } 2 \text {. Post neutralized slides are scanned again to monitor } \\
\text { for remaining autofluorescence. 3. Slides are assayed with fluorescently labeled lectins: } \\
\text { WGA-Texas Red (EY Laboratories) and ConA-FITC (EY Laboratories). }\end{array}$ \\
\hline \multicolumn{2}{|c|}{ 6. Detector and Data Processing } \\
\hline $\begin{array}{l}\text { Scanning } \\
\text { hardware }\end{array}$ & ProScan Array 4 laser (488 nM, 532 nM, 595 nM, 647 nM) scanner (Perkin Elmer). \\
\hline $\begin{array}{l}\text { Scanner } \\
\text { settings }\end{array}$ & $\begin{array}{l}\text { Scanning resolution: } 10 \mu \mathrm{M} \\
\text { Laser channel: } 488 \mathrm{nM} \text { excitation / } 520 \mathrm{nM} \text { emission filter. } \\
\text { PMT: } 70 \% \text { gain } \\
\text { Scan powers: } 100 \% \text { laser power. }\end{array}$ \\
\hline $\begin{array}{l}\text { Image analysis } \\
\text { software }\end{array}$ & ScanArray Express (Perkin Elmer). \\
\hline
\end{tabular}




\begin{tabular}{|l|l|}
\hline $\begin{array}{l}\text { Data } \\
\text { processing }\end{array}$ & Data was exported as a CSV file and exported to Microsoft Excel. \\
\hline 7. Glycan Microarray Data Presentation \\
\hline $\begin{array}{l}\text { Data } \\
\text { presentation }\end{array}$ & $\begin{array}{l}\text { Data is presented as yes/no binding in Figure 1. Data including glycan identification and } \\
\text { analysed values of above background binding (see 8. below) are presented in } \\
\text { Supplementary Table 1. Data presented is in fold-change, which is calculated by the } \\
\text { mean relative fluorescent value of 4 replicate spots divided by the mean background, } \\
\text { defined as the average background of negative control spots plus 3 standard deviations. }\end{array}$ \\
\hline $\begin{array}{l}\text { 8. Interpretation and Conclusion from Microarray Data } \\
\text { interpretation }\end{array}$ & $\begin{array}{l}\text { We only use glycan arrays as a yes/no binding tool. Due to this, we look only at binding } \\
\text { that is unambiguously above background vs lack of binding above background. Average } \\
\text { background }+3 x \text { standard deviation of the background of 20 sets of } 4 \text { spots of } \\
\text { DMF:DMSO only spots is applied to determine if binding observed is significantly } \\
\text { above background. Only spots with values equal to or greater than this value were } \\
\text { considered as binding from data of any tested slide. These values are slide dependent. }\end{array}$ \\
\hline Conclusions & $\begin{array}{l}\text { Glycan binding profile of the two species of Plasmodium sporozoites were different, } \\
\text { and some of the differences could be attributed to host specificity ( } \alpha \text { Gal Epitope). }\end{array}$ \\
\hline
\end{tabular}


(A)

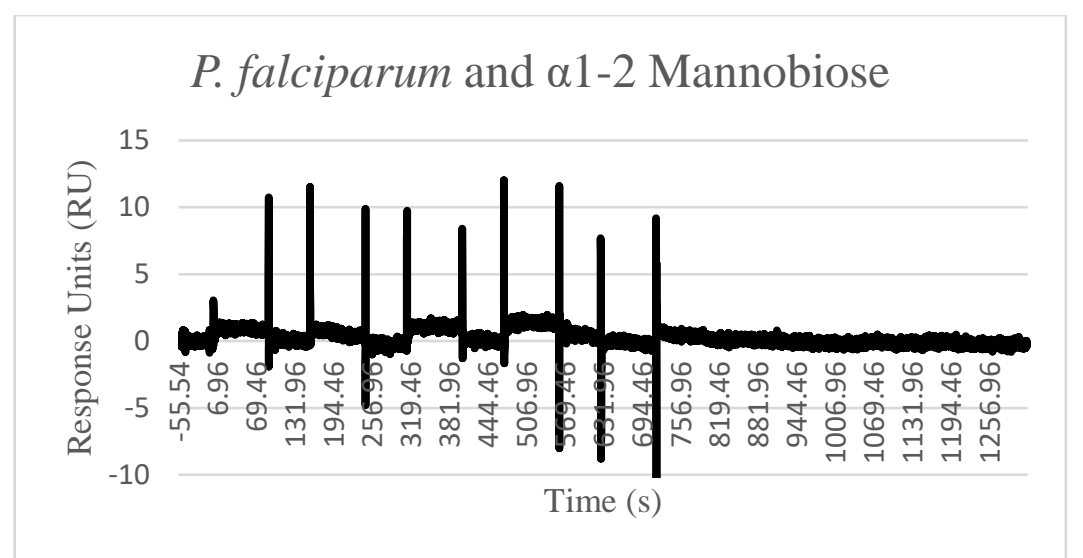

(B)

(C)
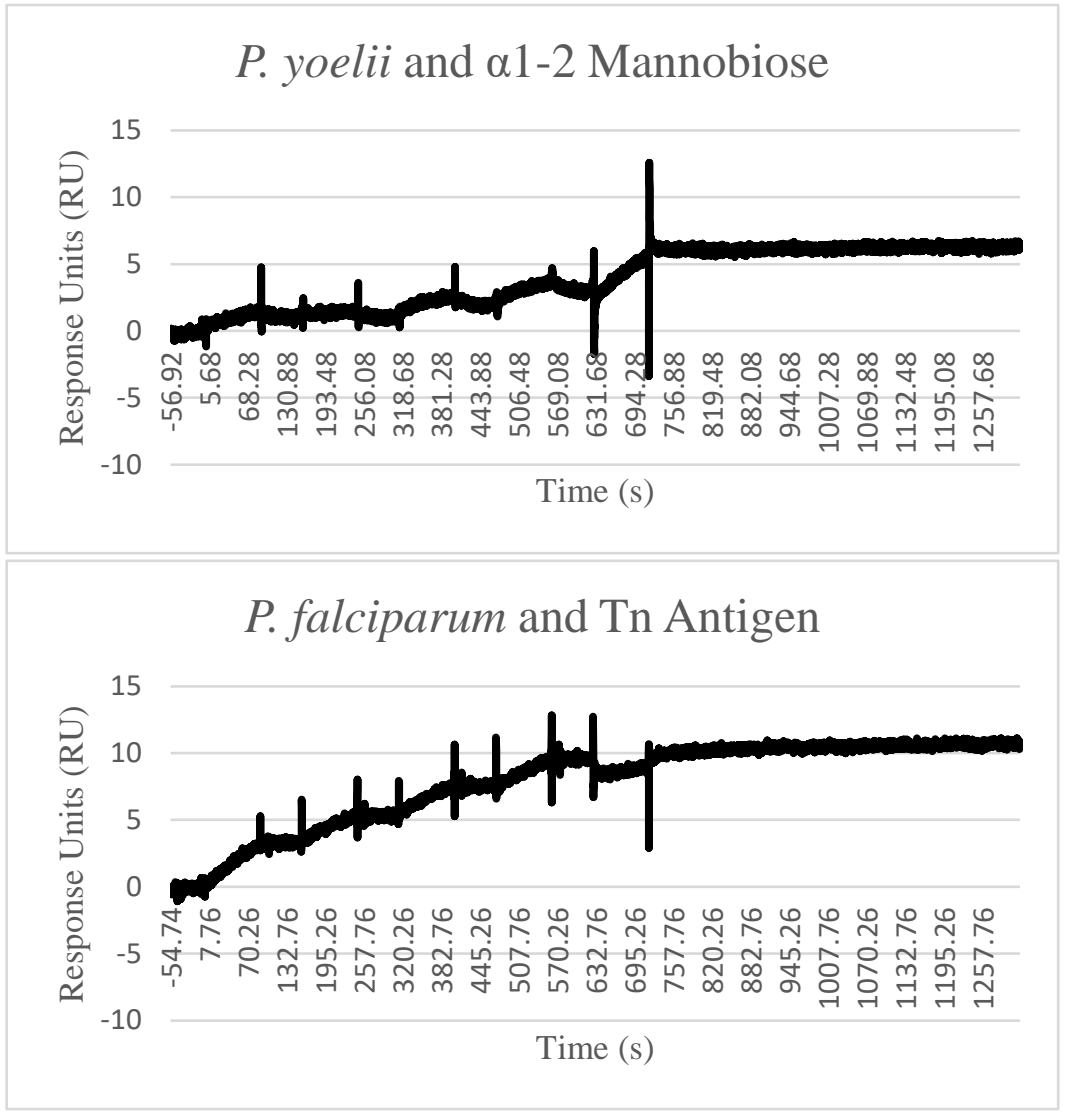

(D)

\section{P. yoelii and Tn Antigen}

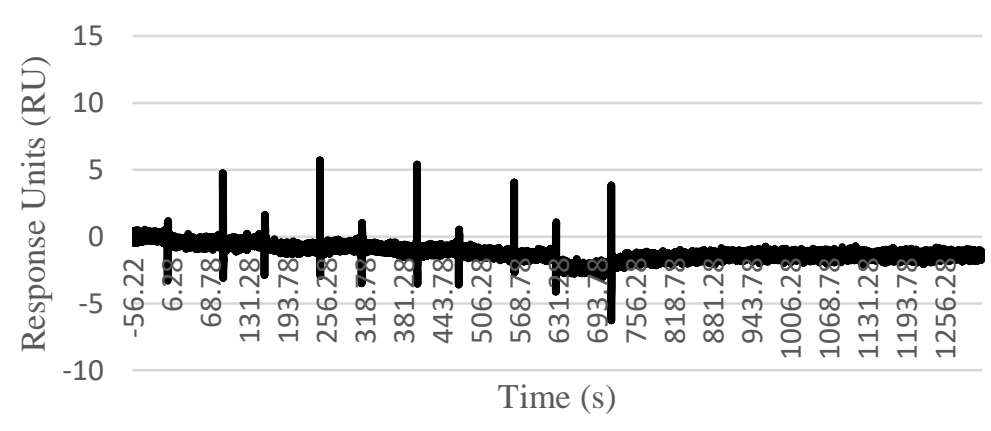


(E)

P. falciparum and 3-O-Su GalNAc

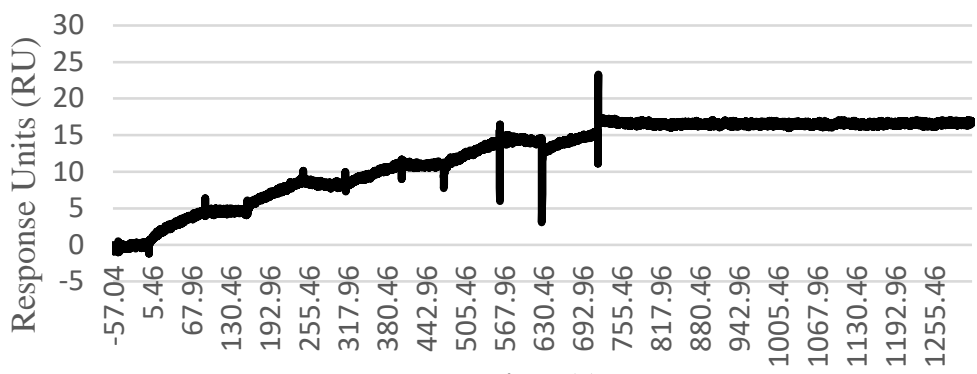

Time (s)

(F)

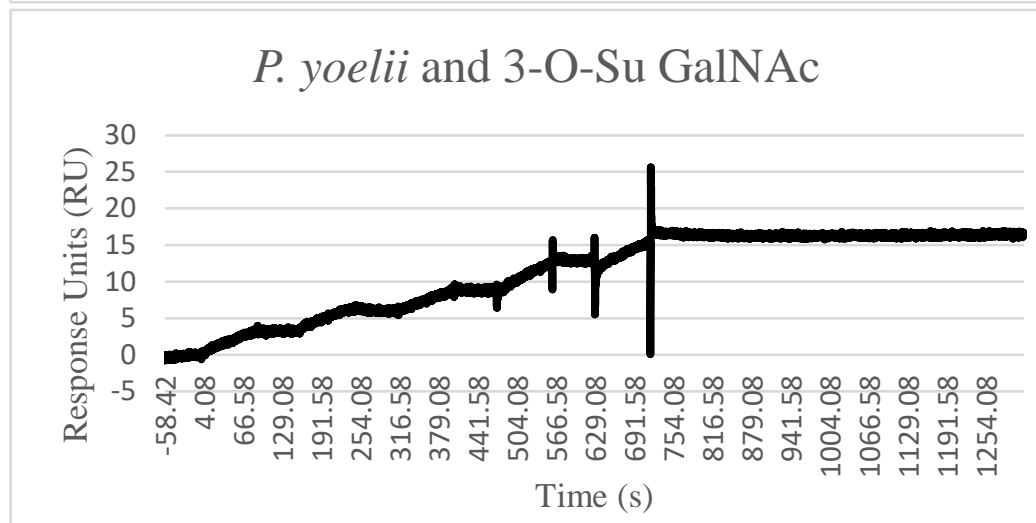

(G)

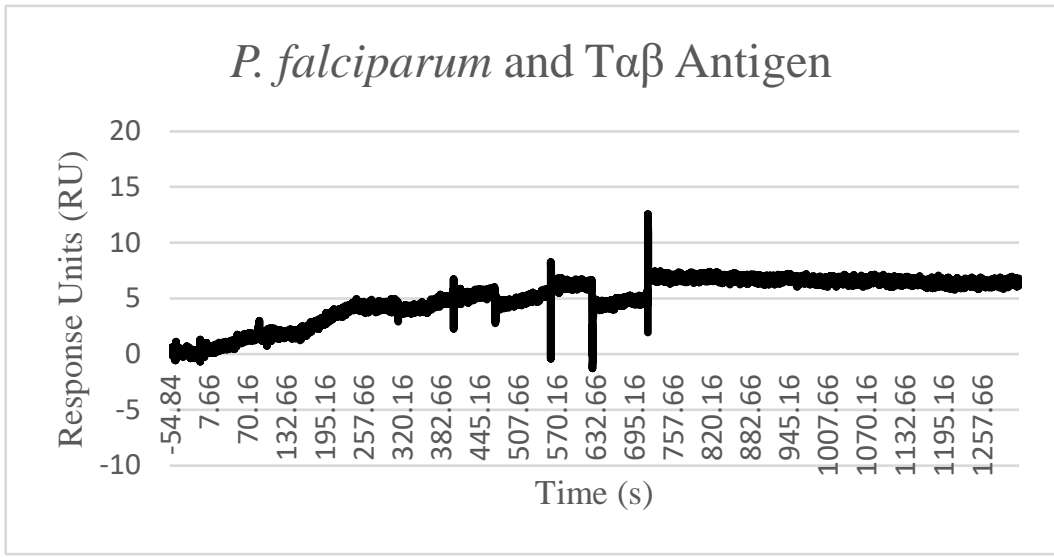

(H)

P. yoelii and T $\alpha \beta$ Antigen

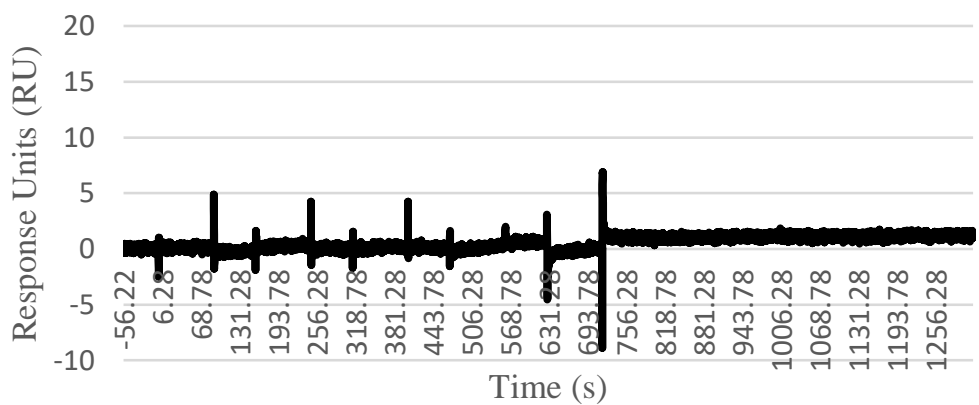


(I)

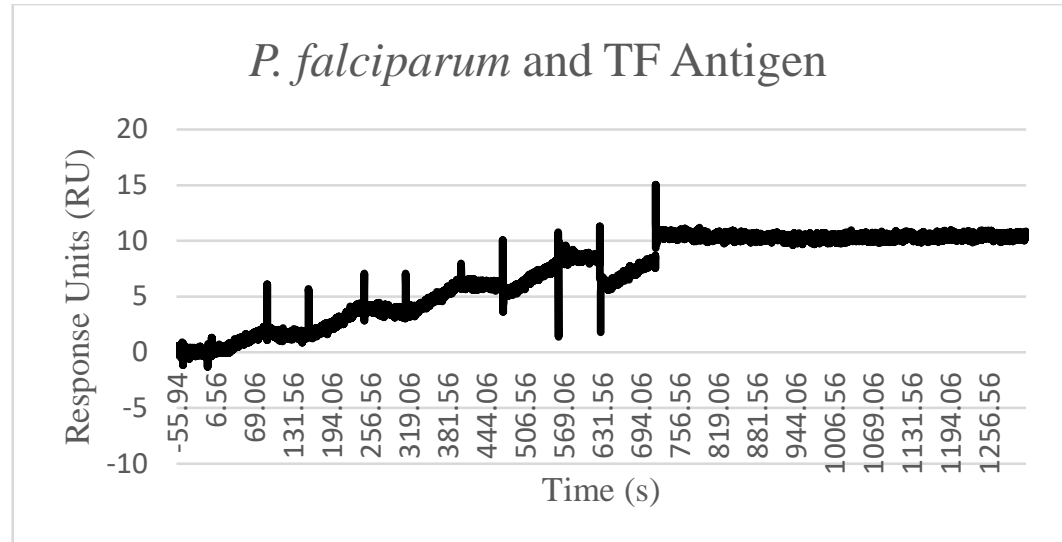

(J)

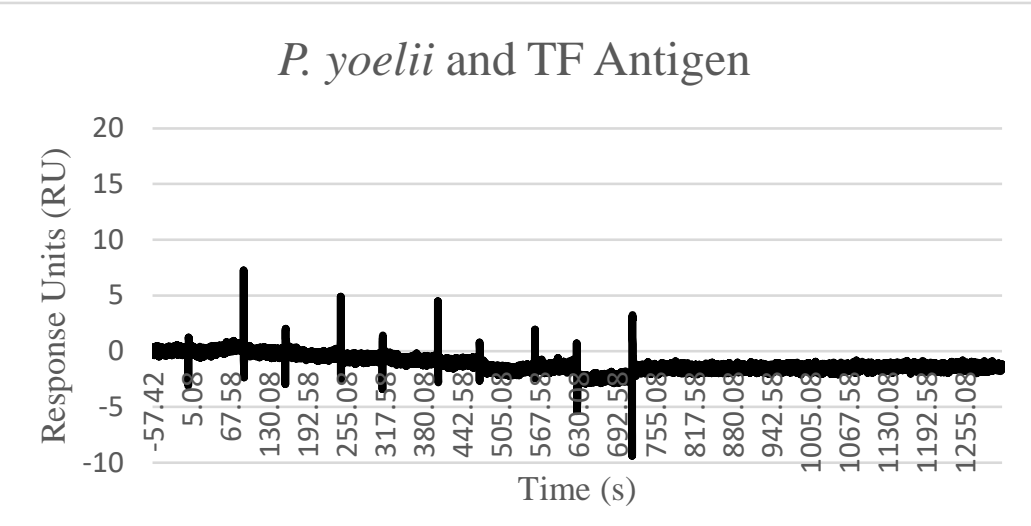

(K)

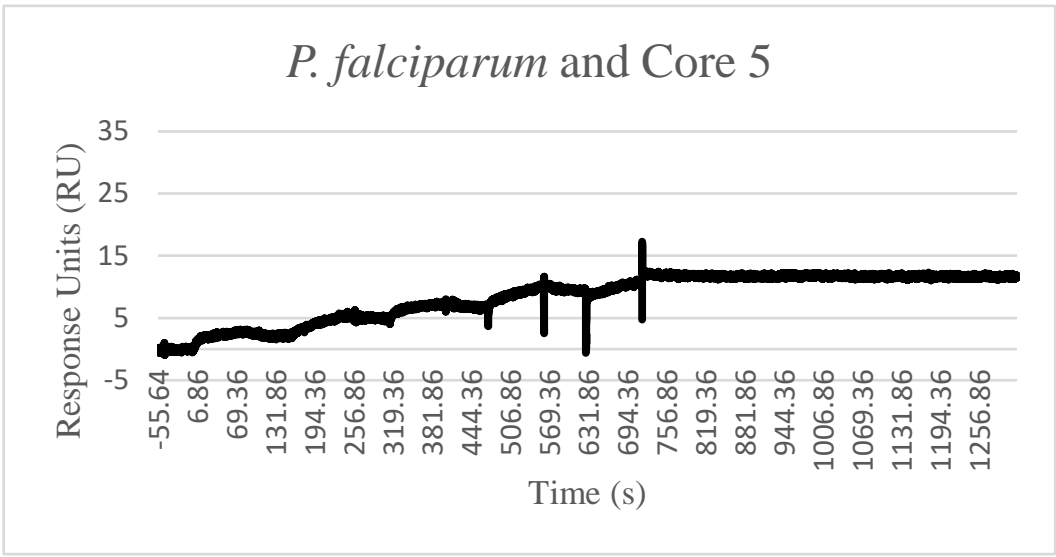

(L)

\section{P. yoelii and Core 5}

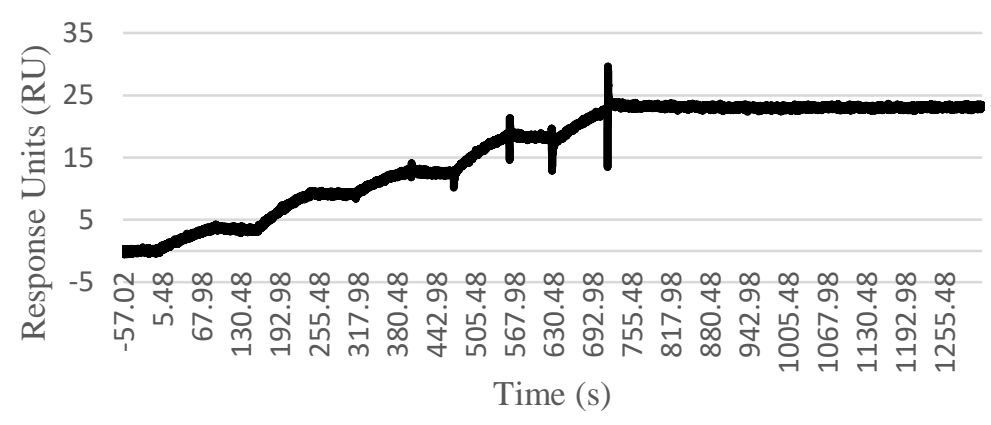


(M)

$P$. falciparum and Lacto-N-Biose I

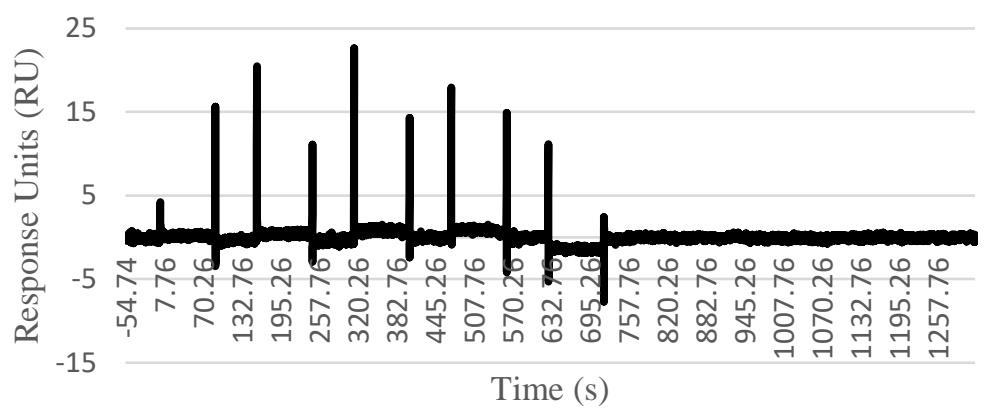

(N)

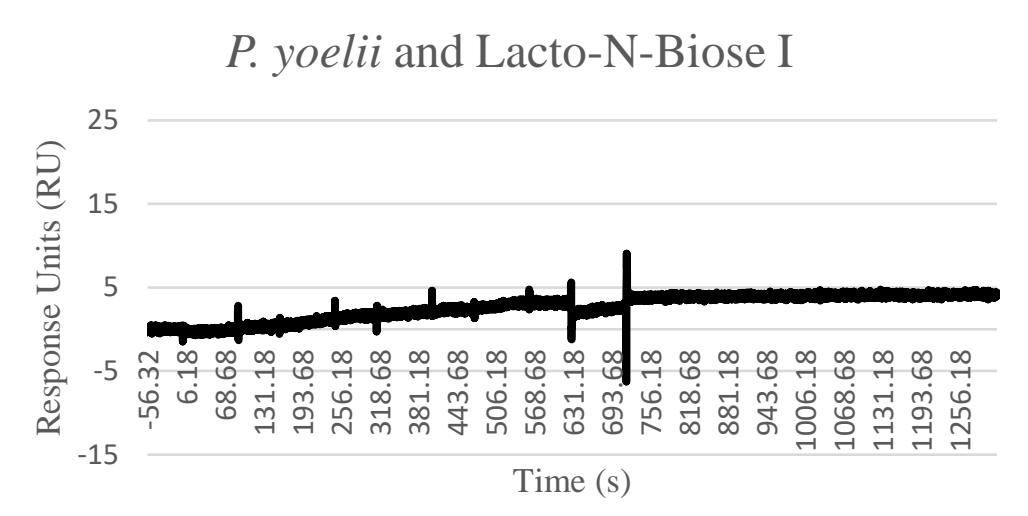

(O)

(P)
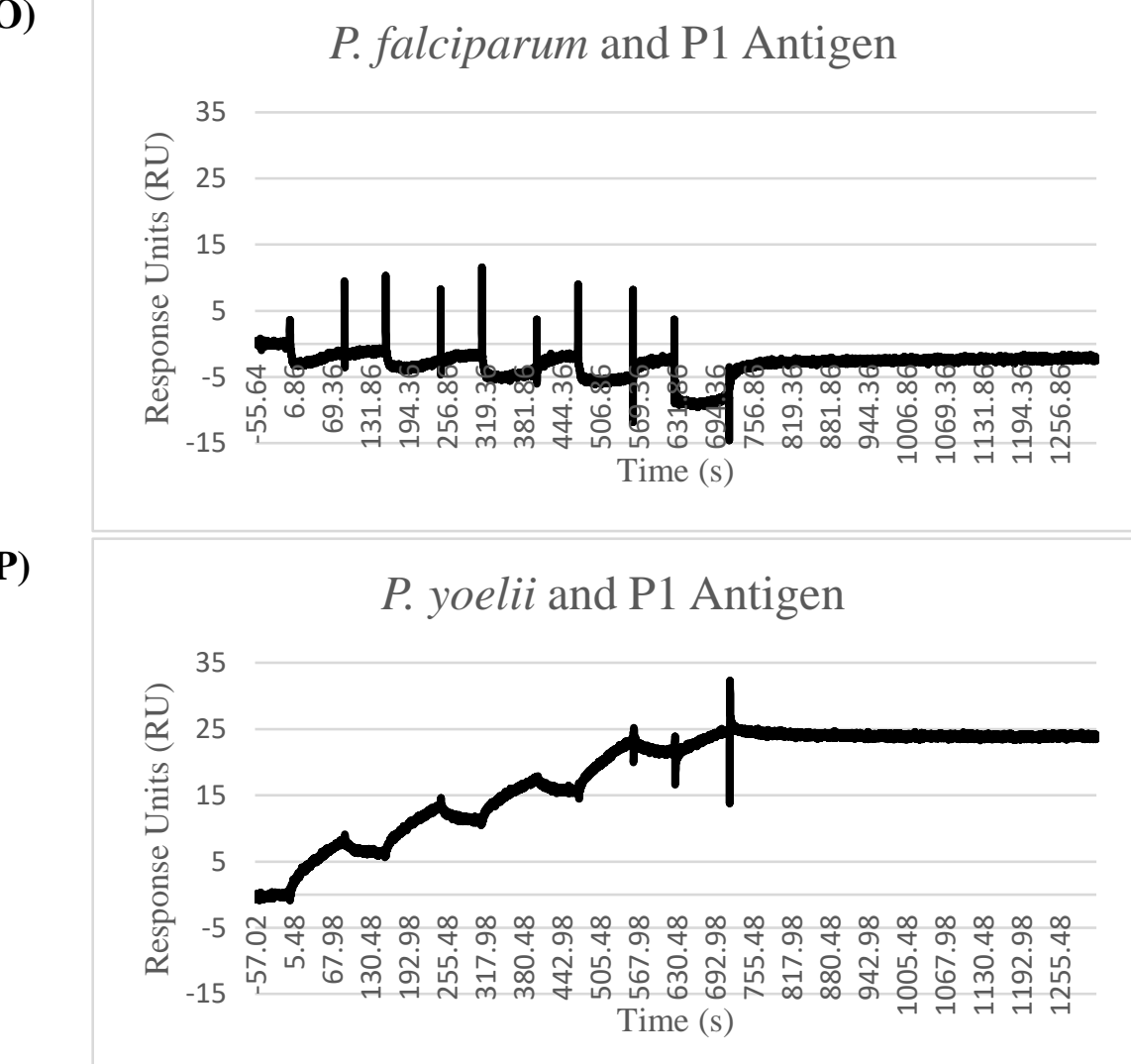
(Q)

(R)

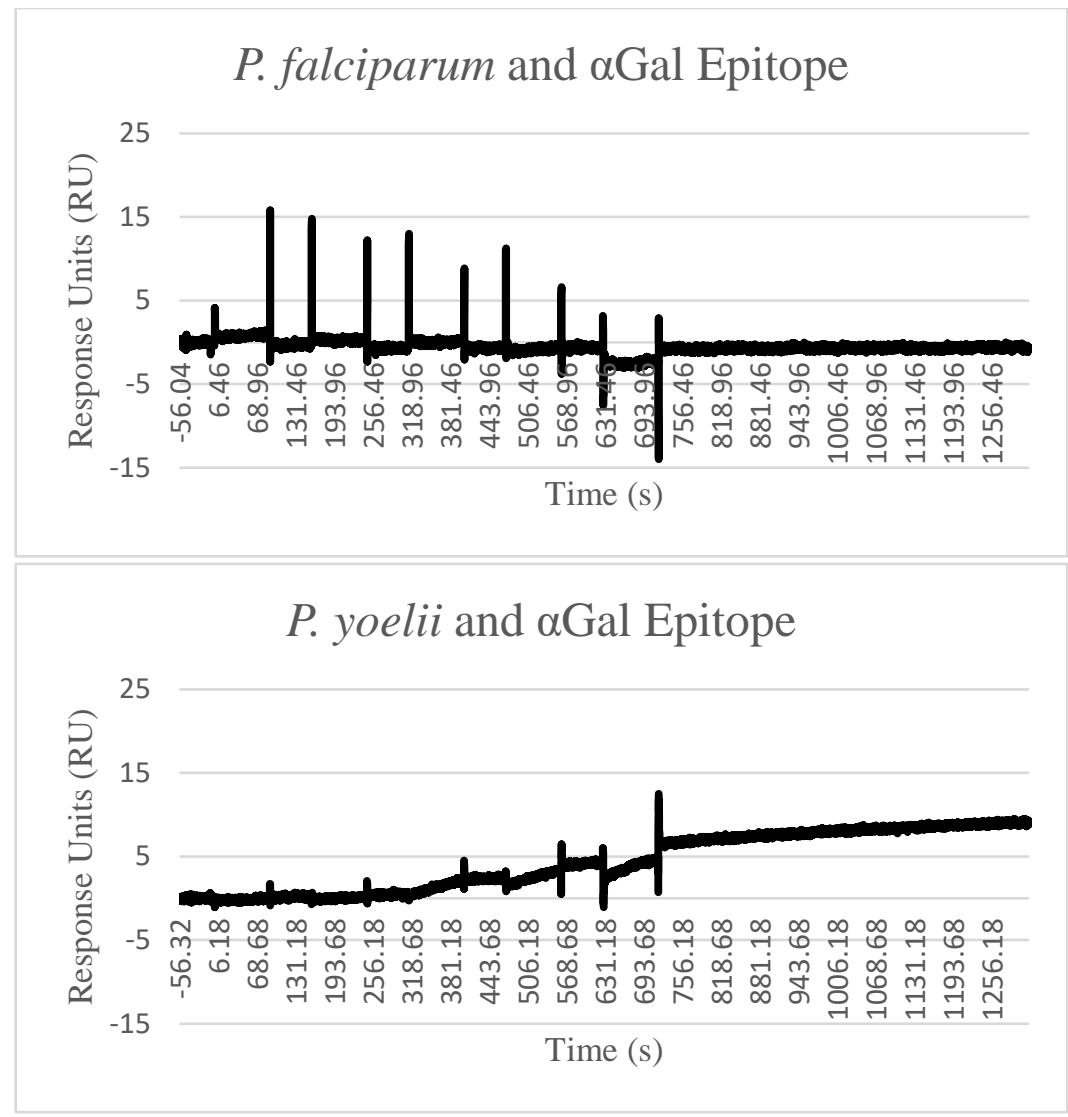

Figure S1. SPR Sensorgrams from Affinity Analysis of Sporozoites with Various

Glycans. The sensorgrams presented are a representative of duplicate data. Sensorgrams show the five dilutions of glycan injected, each dilution for $90 \mathrm{sec}$, over the flow cells containing either immobilized $P$. falciparum or $P$. yoelii with a dissociation time of $600 \mathrm{sec}$ after the final injection of glycan. (A) P. falciparum with $\alpha 1-2$ Mannobiose (Man $\alpha 1-2 M a n$ ). (B) P. yoelii with $\alpha$ 1-2 Mannobiose (Man $\alpha 1-2 M a n)$. (C) P. falciparum with Tn Antigen (GalNAc $\alpha$-Serine). (D) P. yoelii with Tn Antigen (GalNAc $\alpha$-Serine). (E) P. falciparum with O-3-Su GalNAc. (F) P. yoelii with O-3-Su GalNAc. (G) P. falciparum with T $\alpha \beta$ Antigen (Gal $\alpha 1-3$ GalNAc $\beta$ ). (H) P. yoelii with T $\alpha \beta$ Antigen (Gal $\alpha 1-3$ GalNAc $\beta$ ). (I) P. falciparum with TF Antigen (Gal $\beta 1$-3GalNAc $\beta$ ). (J) P. yoelii with TF Antigen (Gal $\beta 1$-3GalNAc $\beta$ ). (K) $P$. falciparum with Core 5 (GalNAc $\alpha 1-3$ GalNAc). (L) P. yoelii with Core 5 (GalNAc 1 13GalNAc). (M) P. falciparum with Lacto-N-Biose I (Galß1-3GlcNAc). (N) P. yoelii with Lacto-N-Biose I (Gal $\beta 1$-3GlcNAc). (O) P. falciparum with P1 Antigen (Gal $\alpha 1-4 \mathrm{Gal} \beta 1$ 4GlcNAc). (P) $P$. yoelii with P1 Antigen (Gala1-4Galß1-4GlcNAc). (Q) P. falciparum with $\alpha$ Gal Epitope (Gal $\alpha 1-3 \mathrm{Gal} \beta 1-4 \mathrm{GlcNAc}$ ). (R) P. yoelii with $\alpha \mathrm{Gal}$ Epitope (Gal $\alpha 1-3 \mathrm{Gal} \beta 1$ 4GlcNAc). 


\section{References}

1. Liu, Y.; McBride, R.; Stoll, M.; Palma, A. S.; Silva, L.; Agravat, S.; AokiKinoshita, K. F.; Campbell, M. P.; Costello, C. E.; Dell, A.; Haslam, S. M.; Karlsson, N. G.; Khoo, K. H.; Kolarich, D.; Novotny, M. V.; Packer, N. H.; Ranzinger, R.; Rapp, E.; Rudd, P. M.; Struwe, W. B.; Tiemeyer, M.; Wells, L.; York, W. S.; Zaia, J.; Kettner, C.; Paulson, J. C.; Feizi, T.; Smith, D. F., The minimum information required for a glycomics experiment (MIRAGE) project: improving the standards for reporting glycan microarraybased data. Glycobiology 2017, 27, 280-284. DOI: 10.1093/glycob/cww118.

2. Waespy, M.; Gbem, T. T.; Elenschneider, L.; Jeck, A. P.; Day, C. J.; HartleyTassell, L.; Bovin, N.; Tiralongo, J.; Haselhorst, T.; Kelm, S., Carbohydrate recognition specificity of trans-sialidase lectin domain from Trypanosoma congolense. PLoS Negl Trop Dis 2015, 9, e0004120. DOI: 10.1371/journal.pntd.0004120.

3. Day, C. J.; Tiralongo, J.; Hartnell, R. D.; Logue, C. A.; Wilson, J. C.; von Itzstein, M.; Korolik, V., Differential carbohydrate recognition by Campylobacter jejuni strain 11168: influences of temperature and growth conditions. PLoS One 2009, 4, e4927. DOI: 10.1371/journal.pone.0004927.

4. Blixt, O.; Head, S.; Mondala, T.; Scanlan, C.; Huflejt, M. E.; Alvarez, R.; Bryan, M. C.; Fazio, F.; Calarese, D.; Stevens, J.; Razi, N.; Stevens, D. J.; Skehel, J. J.; van Die, I.; Burton, D. R.; Wilson, I. A.; Cummings, R.; Bovin, N.; Wong, C. H.; Paulson, J. C., Printed covalent glycan array for ligand profiling of diverse glycan binding proteins. Proc Natl Acad Sci U S A 2004, 101, 17033-17038. DOI: 10.1073/pnas.0407902101.

5. Huflejt, M. E.; Vuskovic, M.; Vasiliu, D.; Xu, H.; Obukhova, P.; Shilova, N.; Tuzikov, A.; Galanina, O.; Arun, B.; Lu, K.; Bovin, N., Anti-carbohydrate antibodies of normal sera: findings, surprises and challenges. Mol Immunol 2009, 46, 3037-3049. DOI: 10.1016/j.molimm.2009.06.010. 